\title{
L'AEQUITAS ROMANA: UNA NOZIONE IN CERCA DI EQUILIBRIO
}

\author{
DARIO MANTOVANI (*)
}

SunTo. - L'aequitas del Corpus Iuris Civilis (e della retorica) ha costituito la matrice della nozione di equità, sia in ambito giuridico, sia in altre scienze sociali; il suo contenuto è tuttavia molto controverso. Il presente studio propone di considerare l'aequitas - più che una nozione unitaria o viceversa vaga e indefinita, secondo i due poli entro $i$ quali oscillano spesso le ricostruzioni moderne - una nozione storicamente complessa, che senza abbandonare il nucleo semantico originario, vi ha aggiunto significati. Lo studio muove da una disamina dell'aequum, dalle prime attestazioni in età repubblicana, fino alla riflessione ciceroniana: se ne ricava che era un concetto già ben radicato ed elaborato dalla cultura romana prima della riformulazione nei termini della filosofia greca, da cui uscirà tuttavia notevolmente arricchito, al limite della sua tenuta. Particolare attenzione è dedicata, sotto questo profilo, a distinguere l'aequitas dall'epieikeia teorizzata da Aristotele. Si verificano inoltre gli esiti di questo tragitto nel pensiero dei giuristi romani. Se ne conclude che l'aequitas è, nella sua prima accezione, un atteggiamento di decisione che ha per criterio l'aequum, ossia l'uguale: conclusione confermata dalle rappresentazioni iconografiche. L'aequitas si iscrive però - almeno dall'epoca tardo-repubblicana - in una più complessiva visione dell'uomo e dei suoi rapporti con gli altri in società, ossia in un'antropologia politica, che ne amplia e precisa i significati (e senza la quale può invece dare l'impressione di essere una nozione vaga).

$$
* * *
$$

ABSTRACT. - The term aequitas in the Corpus Iuris Civilis (and in rhetoric) forms the origin of the notion of equity, both in the field of law and in other social sciences. Its contents are, however, very contentious. Rather than following the two extremes in modern reconstructions, which consider aequitas either as unitary or as a vague and indefinite notion, this paper considers aequitas as an historically complex notion that

(*) Istituto Lombardo Accademia di Scienze e Lettere; Università degli Studi di Pavia, Italia. Project Redhis "Rediscovering the hidden structure. A new appreciation of juristic texts and patterns of thought in Late Antiquity" (ERC AdG 2013).

E-mail: dario.mantovani@unipv.it 
kept its original semantic nucleus, but saw the addition of further meanings. This paper is based on a close examination of aequum, from the earliest attestations in the Republican Age until Cicero. We will see that it was a concept already profoundly rooted and elaborated in Roman culture before its reformulation in terms of Greek philosophy, which considerably enriched it, exploring its limits. In this respect, particular attention is paid to distinguishing aequitas from epieikeia as theorized by Aristotle. This paper examines then the development of the concept in the thought of the Roman jurists. As a whole, this study shows that aequitas involves, at its core, an attitude towards decisions that is founded on the criterion of the aequum, i.e. the "equal"; this conclusion is confirmed by iconographic evidence. Yet, at least from the Late Republic, aequitas is part of a wider conception of man and his relations to others in society, i.e. it is part of a political anthropology that expands and articulates the meanings of aequitas (and without which it might give the impression of being a vague notion).

\section{PREMESSE}

L'aequitas romana è, lessicalmente e concettualmente, l'antecedente dell'equità in ambito giuridico. Inoltre, grazie all'influenza che il diritto ha avuto nell'elaborazione moderna delle altre scienze sociali, dall'economia alla sociologia, l'aequitas del Corpus Iuris Civilis (con il contributo della retorica latina e dell'aristotelismo) s'è diffusa per rifrazione in altre discipline ${ }^{1}$. Un'indagine sull'aequitas elaborata a Roma

1 Per fare un esempio, la nota affermazione di Adam Smith sulla retribuzione degli operai è basata su uno dei contenuti fondamentali del concetto romano di aequitas, cioè sul principio secondo cui chi conferisce, a sue spese, un vantaggio deve partecipare a una quota di esso («It is but equity, besides, that they who feed, clothe, and lodge the whole body of the people, should have such a share of the produce of their own labour as to be themselves tolerably well fed, clothed, and lodged»: An inquiry into the nature and causes of the wealth of nations, I, Dublin, 1776, 116). Si noti che l'avverbio besides assegna alla motivazione basata sull'equity un valore indipendente e assoluto, cioè separato dalle ulteriori argomentazioni utilitaristiche svolte da Smith nel medesimo capitolo, tese a dimostrare che il benessere delle classi più povere è vantaggioso per il benessere delle altre classi sociali. Su questo brano, vd. ad es. J.T. Young - B. Gordon, Distributive justice as a normative criterion in Adam Smith's political economy, in History of Political Economy 28,1 (1996) 1-25 e M. Fortier, The culture of equity in Restoration and eighteenth-century Britain and America, Farnham, 2015, 11, che giustamente, tenendo conto delle letture del XVIII secolo britannico, più dell'influenza aristotelica sottolinea quella latina, in particolare del De officiis ciceroniano. 
può dunque costituire un punto di partenza di un qualche interesse per la riflessione "sulle equità" al plurale, cui è dedicato l'insieme del presente volume. La ricognizione sarà qui svolta in prospettiva storica, ricorrendo a materiali sia lessicali sia iconografici.

Non ci si può attendere - è bene premetterlo - che le fonti antiche restituiscano un significato univoco di aequitas (e dell'aggettivo aequus, da cui essa deriva). Proprio perché era un termine valoriale, il suo significato reagiva a contatto con il contesto (ad esempio, in rapporto ai diversi istituti giuridici in relazione ai quali era impiegato). Incide anche la prospettiva degli scrittori antichi: spesso gli interpreti moderni pervengono a ricostruzioni divergenti dell'aequitas perché gli uni si appoggiano alle (rare) definizioni esplicite che di aequitas offrono le fonti ${ }^{2}$, gli altri ricavano invece induttivamente il concetto dagli usi, senza tenere conto - reciprocamente - che si tratta di testimonianze non necessariamente coincidenti già nelle intenzioni degli antichi. Inoltre, come vale per ogni termine assiologico, il significato di aequitas subì un'evoluzione semantica nel corso degli otto secoli circa durante il quale ne sono documentate le occorrenze, dalle prime testimonianze di III-II sec. a.C. fino a Giustiniano nel VI secolo d.C.

Pur con queste cautele di metodo, sarebbe tuttavia eccessivo negare che vi sia stato un nucleo di significato, soprattutto in settori definiti (ad esempio il diritto) e in periodi storici omogenei. Non si capirebbe altrimenti perché gli antichi si azzardassero a dare definizioni dell'aequitas e soprattutto a usarla come argomento di decisioni da motivare e sostenere in contraddittorio: una formula vuota non basta per convincere chi ha interessi contrapposti. Conferma notevole viene dagli studi iconografici relativi alle figurazioni dell'aequitas (si dovrebbe anzi scrivere Aequitas, trattandosi di personificazione), che hanno sperimentato negli ultimi anni un vero e proprio salto di qualità e i cui risultati non sono finora stati utilizzati dagli storici del diritto romano. La stabilità della rappresentazione dell'aequitas (e la sua differenza rispetto alla figurazione della iustitia) indica che i Romani assegnavano alla nozione un contenuto definito, suscettibile appunto di essere tradotto in immagine (e distinto da altri contigui) ${ }^{3}$. Questi risultati danno ragione, in un certo senso, a quel che sostenevano

\footnotetext{
2 Vd. infra p. 22 e p. 44 nt. 69.

3 Vd. infra $\$ 8$.
} 
intuitivamente i giuristi romani, quando esortavano il giudice a tenere l'aequitas davanti agli occhi, il che significa che le attribuivano tratti in qualche modo riconoscibili (aequitatem ... ante oculos habere debet index $)^{4}$.

L'aequitas è insomma una nozione in cerca di equilibrio, fra la vaghezza e l'eccesso di significato, fra l'esaltazione idealizzata e il ripudio. È questo senz'altro un caso in cui la perenne attualità di un concetto rende più difficile avvicinarsi alle testimonianze antiche in modo sufficientemente immune da precomprensioni, che è la condizione indispensabile per farsene istruire. La rifrazione dell'aequitas in molti ambiti della cultura medievale e moderna ne ha aumentato per così dire retrospettivamente la pluralità di accezioni ${ }^{5}$. Più in generale, il fatto che in nome dell'equità continuino a svolgersi dispute di pensiero e confronti di valore (in fondo, lo stesso presente volume collettivo ne è una testimonianza) condiziona l'esame storico, che inclina non di rado verso quella fra le varie accezioni che sembra preferibile in chiave attuale: il passato serve più che altro a legittimare scelte del presente ${ }^{6}$. Così, non di rado chi cerca un significato unitario dei vari impieghi s'espone al rimprovero di giusnaturalismo, mentre chi rinuncia a trovare un comune denominatore sembra troppo ansioso di mostrarsi in sintonia con l'attuale pluralismo valoriale.

Liberarsi del tutto dalle proprie sensibilità non è possibile: ma almeno si può cercare un antidoto nella lettura ordinata delle fonti e nell'integrazione di documenti di natura diversa.

Cercheremo di offrire alcune coordinate, soffermandoci in particolare sulla storia dell'aequum dalle prime attestazioni in età repub-

4 Ulp. 27 Ad ed. D. 13.4.4.1: nel contesto, il riferimento è al giudice investito dell'actio de eo quod certo loco dari oportet, che consentiva maggiore discrezionalità rispetto alla normale condictio.

5 Fra le tante varianti: aequitas infra legem, praeter legem, contra legem; aequitas Martiniana o bursalis; aequitas imaginaria et crebrina; aequitas Christiana e canonica; equità integrativa o suppletiva, correttiva, sostitutiva: ciascuna di queste accezioni serve a sua volta agli interpreti moderni per rileggere le fonti antiche. Per un eccellente quadro critico, rinvio a A. Donati, Giusnaturalismo e giuspositivismo nella interpretazione della norma giuridica, in Studi in onore di Cesare Massimo Bianca, I, Milano, 2006, 97 177; Id., I valori della codificazione civile, Padova, 2009.

6 Per un esempio recente di attualizzazione, vd. F. Jacques - S. Goyard (éd.), Le droit, le juste, l'équitable, Paris, 2014. 
blicana, fino alla riflessione ciceroniana: se ne ricava che era un concetto già ben radicato nella cultura romana prima della riformulazione nei termini della filosofia greca, da cui uscirà tuttavia notevolmente arricchito, al limite della sua tenuta. Verificheremo poi gli esiti di questo tragitto nel pensiero dei giuristi romani. Concluderemo che l'aequitas è, nella sua prima accezione, un atteggiamento di decisione che ha per criterio l'aequum, ossia l'uguale, e «la cui funzione principale è di assicurare soluzioni conformi alla giustizia distributiva o commutativa» ${ }^{7}$. Essa si iscrive però - almeno dall'epoca tardo-repubblicana in una più complessiva visione dell'uomo e dei suoi rapporti con gli altri in società, ossia in un'antropologia politica, che ne amplia e precisa i significati (e senza la quale può invece dare l'impressione di essere una nozione vaga).

In sintesi, più che una nozione unitaria o viceversa indefinita, l'aequitas è una nozione storicamente complessa, che senza abbandonare il nucleo semantico originario, vi ha aggiunto significati.

7 P. Voci, Ars boni et aequi, in Id., Ultimi studi di diritto romano, a c. di R. Astolfi, Napoli, 2008, 289-313, spec. 304: l'intero saggio offre, pur nella sua sinteticità, utili riferimenti alle fonti e interpretazioni persuasive. Nell'immensa bibliografia, fra gli studi più recenti segnalo anche: A. Biscardi, On aequitas and epieikeia, in A.M. Rabello (ed.), Aequitas and equity: equity in civil law and mixed jurisdictions, Jerusalem, 1997, 1-11; M. Humbert, The concept of equity in the Corpus Iuris Civilis and its interpretation by Pothier, ibid., 29-47; M. Talamanca, L'Aequitas nelle costituzioni imperiali del periodo epiclassico, in G. Santucci (a c. di), Aequitas. Giornate in memoria di Paolo Silli, Padova, 2006, 53-273; M. Bretone, Labeone e l'ordine della natura, D. Mantovani - A. Schiavone (a c. di), Testi e problemi del giusnaturalismo romano, Pavia, 2007, 249-269; W. Waldstein, Equità e ragione naturale nel pensiero giuridico del I secolo d.C., ibid., 299322; M. Humbert, Equité et raison naturelle dans les oewvres de Celse et de Julien, ibid., 419-473; J.F. Stagl, Die Ausgleichung von Vorteil und Nachteil als Inhalt klassischer aequitas, ibid., 675-713; L. Ostwaldt, Aequitas und Justitia. Ibre Ikonographie in Antike und Früher Neuzeit, Magdeburg, 2009, spec. 1-92, pregevole indagine iconografica e concettuale, che contiene importanti precisazioni anche per il diritto romano; $\mathrm{M}$. Bretone, L'equità controluce, in Id., Soliloquio sul diritto antico. La filosofia di una tecnica, Lecce, 2013, 47-56; W. Waldstein, Ist das suum cuique eine Leerformel?, in Id., Vera philosophia. Scritti romanistici scelti, Napoli, 2013, 169-205; T. Finkenauer, Iustitia und iustus bei den römischen Juristen, in Fundamina 20 (2014) 287-300. Altra bibl. in L. Solidoro Maruotti, Tra morale e diritto. Gli itinerari dell'aequitas. Lezioni, Torino, 2013. 


\section{AEQUUS-AEQUITAS: INTRODUZIONE ALLA PAROLA}

L'equità delle lingue moderne (equity, equité, equidad) ha la sua matrice nel lat. aequitas, sostantivo che indica la "condizione di essere aequus". L'aggettivo aequus (-a/-um) ha preceduto il sostantivo astratto ed è già impiegato nei primi autori di cui ci sia giunta una sufficiente documentazione, come Plauto, Ennio, Catone e Terenzio, fra la fine del III e la metà del II secolo a.C. ${ }^{8}$. Aequus (aggettivo per il quale non è stata identificata un'etimologia sicura, come per la maggior parte delle parole a dittongo in -ae- $)^{9}$, significa "uniforme", "piano" in senso orizzontale, "che non presenta differenze". La prossimità semantica fra lat. aequus e gr. "̂́os ("uguale") è ben documentata; ad es., la frase di Sallustio (Iug. 42) in aequum locum deducere ("scendere

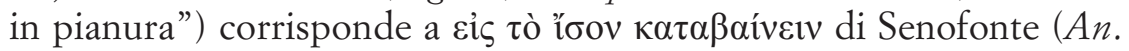
4.6.18) ${ }^{10}$.

Più che per gli usi propri, l'aggettivo aequus è interessante per la sua produttività metaforica - innescata dall'idea di "uguaglianza", "simmetria" - che ha avuto modo di manifestarsi in molti ambiti.

In ambito morale, aequus "che non pende da alcuna parte" significa "imparziale, equilibrato". S'incontra così l'aequus index ${ }^{11}$ (equidistante rispetto alle parti) ${ }^{12}$ e l'aequus animus, equilibrato e capace di

8 Vd. TLL, I, 1028,64 - 1046,65, s.v. aequus. Aequitas è sostantivo attestato in letteratura a partire dalla Rhetorica ad Herennium e da Cicerone (TLL, I, 1013,73 1017,2, s.v. aequitas); tuttavia, nella forma *Aecetia compare già in una coppa di Vulci del 280 a.C. circa (vd. infra, nt. 55). Questa precoce emersione dell' astratto è da tenere in considerazione quando si traccia la storia di questa nozione: troppo spesso si ritiene che lo sviluppo del concetto sia avvenuto solo in età ciceroniana.

9 A. Ernout - A. Meillet, Dictionnaire étymologique de la langue latine, rist. Paris, 1994, 11.

10 Vd. TLL, I, 1029.5, s.v. aequus.

11 Ad es. Cic. II In Verr. 2.72: erat in consilio etiam aequissimus iudex; Liv. 42.41.1: eventus belli velut aecus iudex; Ov. Trist. 1.1.45: iudex mirabitur aequus; CIL XI 6173 lin. 15: non aequo iudice Fato.

12 È facile lo slittamento verso la sostanza della decisione: Ter. Heaut. 213-215: Quam iniqui sunt patres in omnis adulescentis iudices! / Qui aequom esse censent nos a pueris ilico nasci senes / neque illarum adfinis esse rerum, quas fert adulescentia («Che giudici iniqui sono i padri verso tutti gli adolescenti; pensano che dovremmo nascere subito vecchi fin da bambini, e non dovremmo essere attratti da nessuna delle cose che comporta l'adolescenza»). Significativo per il nesso che stabilisce fra equità del giudice 
sopportare i rovesci ${ }^{13}$ : un accostamento, quest'ultimo, talmente fortunato da avere dato luogo al sostantivo aequanimitas.

Una trasposizione simbolica particolarmente efficace si è verificata nel campo della misurazione, perché aequus esprime bene la relazione di corrispondenza fra un oggetto e un altro. Così, i (contrap)pesi usati nelle stadere o i recipienti per liquidi o solidi sono detti equi o iniqui a seconda che equivalgano o meno alla quantità pesata o misurata. Questo simbolismo è tanto persistente che l'Aequitas ipostatizzata come virtù degli imperatori romani si trova associata alle Mensurae: una iscrizione di Philippi (riferita a un imperatore non meglio identificato, fra I e III secolo) ricorda, quasi a chiudere il cerchio di questa relazione fra astratto e concreto, che il bronzo necessario a innalzare il monumento all'Aequitas Augusti e alle Mensurae era stato ricavato dagli edili ex mensuris iniquis, cioè fondendo contrappesi fallaci in bronzo ${ }^{14}$.

e bilancia Min. Fel. 5.1: ut libram teneas aequissimi iudicis. Altre iuncturae sono iudicium aequum (ad es. Cic. Clu. 5: illa definitio iudiciorum aequorum quae nobis a maioribus tradita est retineatur, ut in indiciis et sine invidia culpa plectatur et sine culpa invidia ponatur; «sia mantenuto quell'ordinamento dei processi equi che ci è stato tramandato dagli antichi, così che nei processi la colpa sia punita senza risentimenti e dove non c'è colpa si abbandoni l'ostilità») e aequus arbiter (Plaut. Cas. 966: nunc ego tecum aequom arbitrum extra considium captavero; Trucul. 629).

13 Ad es. Plaut. Rud. 402: animus aequos optimum est aerumnae condimentum; Ter. Ad. 738: animo aequo fero; Cic. Att. 15.25: meus animus est aequus, sic tamen, ut si nibil offensionis sit.

14 AE 1935, 49 = P. Pilhofer, Philippi II. Katalog der Inschriften von Philippi, Tübingen, 2000, 265-267, nr. 249: Aequitatem Augusti / et Mensuras / M(arcus) Cornelius P(ubli) f(ilius) Vol(tinia) Niger / P(ublius) Valerius P(ubli) f(ilius) Vol(tinia) Niger / aed(iles) d(e) s(ua) p(ecunia) f(aciendum) c(uraverunt); in id opus co(n)iectum est ex mensuris / iniquis aeris p(ondos) XXXXIIII. La distruzione (non sempre per il riuso) di pesi o misure inique è attestata ripetutamente: vd. Ulp. 32 Ad ed. D. 19.2.13.8: Si quis mensuras conduxerit easque magistratus frangi iusserit, si quidem iniquae fuerunt, Sabinus distinguit, utrum scit conductor an non: si scit, esse ex locato actionem, si minus, non. Quod si aequae sunt, ita demum eum teneri, si culpa eius id fecit aedilis ( Se uno ha preso in conduzione i pesi e il magistrato ha ordinato che fossero distrutti, se in effetti erano iniqui, Sabino distingue a seconda che il conduttore lo sappia o meno; se lo sa, c'è l'azione contro di lui in base alla locazione; se non lo sapeva, non c'è. Se invece i pesi erano equi, risponde solo se l'edile ha agito così per sua colpa»); Pers. Sat. 1.129-130: Sese aliquem credens Italo quod honore supinus / fregerit beminas Arreti aedilis iniquas («si crede qualcuno, perché rivestito di una magistratura italica, come edile ad Arezzo ha distrutto dei mezzi pesi»). 
Coerentemente, come vedremo meglio più avanti, l'attributo che contraddistingue iconograficamente l'aequitas sulle monete è la bilancia ${ }^{15}$.

In tutte le sue applicazioni, dunque, aequus è accompagnato da un carico semantico legato all'idea di uguaglianza, di corrispondenza, di proporzione, di equilibrio. È un punto di riferimento da mantenere presente nell'interpretazione dei suoi pur vari usi ${ }^{16}$. È anche il nocciolo su cui concordano le rare definizioni di aequitas che ci sono pervenute dall'antichità latina, di Seneca (Ep. 30.11): prima autem pars est aequitatis aequalitas e di Isidoro di Siviglia (Orig. 10.7): aequus est secundum naturam iustus dictus, ab aequalitate, quod sit aequalis, unde et aequitas appellata, ab aequalitate quadam scilicet («equo è detto il giusto secondo natura, da 'uguaglianza', perché è uguale, da ciò viene il termine equità, ossia da una sorta di uguaglianza»). ${ }^{17}$

\section{AEQUUM EST, UN VALORE GIURIDICO ROMANO}

Se la produttività metaforica ne fa un aggettivo ben diffuso, sono i discorsi attinenti al diritto quelli che, nell'antichità romana, più frequentemente sfruttano il valore semantico dell'eguaglianza insito in aequus. Sul piano della tradizione successiva, sono proprio i discorsi di questo genere che hanno direttamente influenzato la riflessione giuridica moderna e si sono poi diffusi in altre discipline. In primo luogo attraverso il Corpus Iuris Civilis, poi attraverso i testi retorici, con Cicerone in primo piano (e, come vedremo, con il contributo dell'Etica Nicomachea di

15 Ovviamente, se i pesi sono equi, a sua volta la bilancia sarà in piano, aequa lanx. Sulla bilancia, vd. altri dettagli infra, $\$ 8$. La relazione fra iustitia e tutela della equa pesatura è dichiarata da Apul. Plat. dogm. 2.7: iustitia (...) ponderum mensurarumque custos.

16 Bretone, L'equità controluce, 48: «aequitas rinvia sempre a un'idea di corrispondenza e di equilibrio, di similitudine o di eguaglianza vera e propria; ma le differenze sono profonde e le sfumature infinite».

17 In quest'ultima definizione, al nocciolo semantico incentrato sull'equaglianza si aggiunge il riferimento giusnaturalistico, secondo una movenza che rappresenta bene le due dimensioni che, come cercherò di mostrare, definiscono l'aequitas romana. Anch'essa incentrata sull'eguaglianza è la definizione di un aspetto dell'aequitas (non dell'aequitas in generale) data da Cic. Top. 23: valeat aequitas, quae paribus in causis paria iura desiderat («si applichi l'equità, che in situazioni eguali desidera discipline eguali»). Su Donat. Ad Ter. Ad. 51-52, vd. infra, nt. 69. 
Aristotele). Non è dunque il valore semantico del latino aequus/aequitas in quanto tale (nelle varie sue occorrenze nelle fonti) il referente della concettualizzazione medievale e moderna, bensì più specificamente il modo in cui il concetto è stato forgiato dalla tradizione giuridica romana e, secondariamente, dalla retorica. Nel nostro breve contributo, cercheremo tuttavia di rintracciare il significato che questi termini ebbero prima di essere utilizzati dai giuristi le cui opere furono poi inserite nel Corpus Iuris Civilis, perché una breve storia semantica e concettuale consente di apprezzare meglio quanto della nozione di aequitas dipenda dall'esperienza e mentalità romana, e quanto da influenze culturali esterne (la ricognizione degli antefatti è necessaria, perché i giuristi le cui opere ci sono note ricevono il termine in una sua accezione già ben forgiata).

A differenza di quel che spesso si ritiene, l'impiego di aequum (esse) in campo giuridico non è successivo all'incontro della giurisprudenza romana con la retorica e la filosofia greca ${ }^{18}$. L'uso di questo schema argomentativo è ben attestato fin dal primo momento in cui la documentazione permette qualche riscontro.

Quando si attribuisce grande spazio all'aequitas ciceroniana si rischia di scambiare la condizione lacunosa della nostra documentazione con i fenomeni storici. Le opere di Cicerone - sia quelle retoriche, sia quelle filosofiche - rappresentano un corpus di testi molto più abbondante rispetto alle fonti giuridiche repubblicane, anteriori a Cicerone stesso, ma anche a lui contemporanee. Per questo, attribuire pressoché esclusivamente alla riflessione ciceroniana lo sviluppo dell'aequitas rischia di condurre a conclusioni inadeguate. Un'esplorazione delle poche fonti disponibili mette in crisi questo assunto: perciò ritengo opportuno insistervi, sia perché fonti importanti sono state finora trascurate, sia perché il fatto che l'aequum emerga già con tratti consolidati in età pre-ciceroniana permette di meglio giudicare l'apporto della filosofia greca, in che

18 «Ein urrömischer Begriff»: così giustamente Ostwaldt, Aequitas und Justitia, 72; anche Voci, Ars boni et aequi, 303: «la nozione del bonum et aequum non poteva pervenire se non dall'esperienza di cose romane, e non poteva essere dovuta a speculazione retorica». Diversamente Solidoro Maruotti, Tra morale e diritto, 49, 172 limita l'esame delle fonti preciceroniane a cinque testimonianze plautine (non discusse analiticamente) che esprimerebbero un richiamo «al concetto morale di aequom», mentre sarebbe stata l'arte retorica e argomentativa, con Cicerone, «a favorire l'introduzione e la diffusione, in Roma, della nozione di aequitas (probabilmente collegata alla dottrina aristotelica dell'epieikeia)». 
misura abbia o non abbia influito sulla nozione romana (e anche di valutare più esattamente l'innegabile apporto di Cicerone stesso).

Iniziamo dalle (poche) fonti giuridiche ${ }^{19}$. Aequom (nella grafia arcaica aiquom) ricorre già, in posizione concettuale saliente, in due dei più antichi senatoconsulti conservati in via epigrafica, nel SCtum de Bacchanalibus del 186 (CIL I ${ }^{2}$ 581, lin. 27: senatus aiquom censuit) e nell'epistola del pretore Lucio Cornelio Lentulo Lupo che riferisce il SCtum de Tiburtibus (CIL I 201, lin. 4: sicut aiquom fuit) ${ }^{20}$. In entrambi i casi, aiquom ha valore politico-giuridico. Simmetricamente, la sentenza emessa nel 117 a.C. dai due arbitri (Quinto e Marco Minucio Rufo) incaricati dal senato di risolvere le controversie fra i Genuates e i Viturii

${ }^{19}$ Fra i giuristi più antichi, aequum compare in Servio Sulpicio Rufo (coetaneo di Cicerone), apud Gai. Inst. 3.149; apud Paul. 9 Ad ed. D. 3.5.20; cfr. Alf. (Serv.?) 2 Dig. D. 44.1.14. Su queste tre testimonianze, e altre in cui il termine non compare, ma che parrebbero rimandare a soluzioni basate sull'equità, vd. Voci, Ars boni et aequi, 300-302. Si noti che sebbene Servio - almeno in queste sparse testimonianze - non usi il sostantivo aequitas, Cicerone ricorre proprio al sostantivo per fare l'elogio funebre di Servio (Phil. 9.10: divina eius ... aequitate explicanda scientia). Anche Gaio Aquilio Gallo, giurista di una generazione precedente, è elogiato da Cicerone come qui iuris civilis rationem numquam ab aequitate seiunxerit (Caec. 78). Ciò implica che l'uso del sostantivo, nella prospettiva di Cicerone, non comporta un cambiamento di fondo, cioè esso non fa che esprimere ciò che già era nella formula aggettivale, tradizionalmente usata dai giuristi.

${ }^{20} \mathrm{Nel}$ primo caso si riferisce alla decisione di fare incidere il testo del SCtum stesso su una tavola di bronzo da parte delle comunità italiche destinatarie (utei boce in tabolam ahenam inceideretis ita senatus aiquom censuit, uteique eam figier ioubeatis, ubei facilumed gnoscier potisit; «il senato ha ritenuto equo che incidiate questo in una tavola di bronzo e che ordiniate che essa sia affissa in luogo ove possa essere conosciuta nel modo più facile»); vd. poi nella Tabula Siarensis (AE 1989, 408, lin. 21-22): item senatum velle atque aequ $(u) m$ censere, riferito anche in questo caso al procedimento di divulgazione del testo. Vi è dunque una relazione fra aequum e conoscenza delle norme. Nel caso del SCtum de Tiburtibus (quod Teiburtes v(erba) f(ecistis) quibusque de rebus vos purgavistis ea senatus / animum advortit ita utei aequom fuit; «Le parole che voi Tiburtini avete pronunciato e le cose di cui vi siete giustificati, il senato le ha ascoltate come era equo»), l'aggettivo sembra riferirsi all'atteggiamento imparziale con il quale il senato ha accolto le giustificazioni degli ambasciatori di Tivoli. Non si comprende perché Solidoro Maruotti, Tra morale e diritto, 72-73 includa questi due testi fra $i$ casi in cui il senato aveva «espresso giudizi di 'iniquità' e 'inattualità' del tradizionale ordinamento civilistico, invitando i magistrati a prendere i provvedimenti idonei all'adeguamento del diritto alle nuove istanze, ai nuovi valori sociali indicati dall'alto consesso e conformi a equità». In nessuno dei due casi il senato tratta di questioni attinenti all'ordinamento civilistico. 
contiene una sorta di clausola di riesame, basata sulla nozione di iniquum: Sei quoi de ea re / iniquom videbitur esse, ad nos adeant primo quoque die ( $« \mathrm{Se}$ a qualcuno sembrerà iniquo qualcosa in relazione a questa causa, si rivolgano a noi nel primo giorno utile»). Aequum si trova inoltre - in traduzione greca, e associato a bonum - nella formula dell'actio iniuriarum attestata da un'epigrafe della metà del II sec. a.C., con funzione che nel contesto è essenzialmente estimatoria ${ }^{21}$.

Nonostante la scarsa disponibilità di fonti giuridiche per l'epoca preciceroniana, che l'espressione aequum (esse), posta a base di una decisione, fosse già tipica e distintiva del linguaggio giuridico è dimostrato anche dal fatto che la si trova stabilmente riflessa nella letteratura coeva, ossia nella commedia (Plauto, Terenzio) e nella tragedia (Ennio, Pacuvio). Così, allude parodisticamente proprio alla solennità senatoria (o al consilium che assisteva i magistrati) Plaut. Poen. 794-795: Nunc ibo, amicos consulam, quo me modo / suspendere aequom censeant potissumum («ora me ne vado a consultare gli amici, in che modo ritengano più equo che mi impicchi»).

Nello stesso solco, la qualificazione aequum (esse) si applica in ambito giurisdizionale, a fronte di richieste (orare/postulare) valutate accoglibili:

Plaut. Cas. 500: aequom oras: abi[s].

Plaut. Cist. 765: aequom postulas.

Pacuv. trag. 32: at aecum et rectum sit, quod postulas ${ }^{22}$.

Non vi è contrasto, bensì convergenza valoriale fra aequum e ius (ovviamente purché si tratti di un bonum ius), come mostra Plaut. Stich. 726: Bonum ius dicis: impetrare oportet qui aequom postulat ${ }^{23}$.

Se vi fosse dubbio sulla consonanza valoriale fra ius atque aecum, Ennio lo elimina con quest'aforisma, nell'Hectoris lytra (XIX 188-189): melius est virtute ius: nam saepe virtutem mali / nanciscuntur: ius atque

21 Sulla formula, vd. infra, p. 28.

22 Altri esempi: Plaut. Men. 153; Rud. 184; Cist. 765; Pers. 587. (per altri esempi analoghi, dove compare il binomio bonum et aequum, vd. infra $\$ 4$ ) Quando diventerà corrente il sostantivo, la trasformazione sarà facile: aequitatem expostulationis tuae (Cic. Ep. 3.7.3).

${ }^{23}$ Il dialogo parodistico prosegue: [SA.]: age ergo observa: si peccassis, multam bic retinebo ilico. / [ST.]: Optumum atque aequissimum oras. Vd. ancora Plaut. Stich. 423: ius et aequum postulas. 
aecum se a malis spernit procul ${ }^{4}$ («il diritto è superiore alla prodezza; infatti spesso i malvagi hanno prodezza, mentre il diritto e l'equo si tiene alla larga dai malvagi»). Tanto più l'accostamento è significativo nel senso di considerare ius e aequum valori consonanti (e non già la testimonianza di un'epoca in cui si sentiva la loro opposizione), in quanto il verso enniano, se non erro, è a sua volta ricalcato nella sua ultima parte da Cic. Leg. 1.48, che lo rende così: etenim omnes viri boni ipsam aequitatem et ius ipsum amant («infatti tutti gli uomini perbene amano l'equità e il diritto di per se stessi»). La convergenza non potrebbe essere più chiara.

Aequum est, già a livello dell'incipiente II secolo d.C., è dunque un tecnicismo del lessico giuridico. Quanto alla sostanza, gli ultimi esempi mostrano che aequum non qualifica un decreto che integri o corregga il ius, ma qualifica il normale ius dicere. Una precisazione può essere opportuna. Ius e aequum non sono certamente sinonimi; altro sarebbe però ritenere che l'aequum rappresenti un'istanza contrapposta al ius civile (i cui vizi si identificano di volta in volta con l'eccessivo formalismo e la prevalenza data alle parole sui contenuti). Vero è solo che, proprio perché si riferiscono a fenomeni diversi (il diritto e l'equo), potevano talvolta esservi delle divergenze: proprio nel momento in cui invocano l'aequitas nella costituzione, interpretazione e applicazione del ius, i Romani dimostrano che essi consideravano l'equità un carattere necessario del ius, non la sua antitesi. Nella loro ideologia, il ius non era bonum se non era aequum.

\section{AEQUUM ET BONUM}

Anche il sintagma aequum et bonum (o aequum bonumque o bonum atque aequum) - che abbiamo già incontrato nella formula dell'actio iniuriarum attestata in greco in un'epigrafe di II secolo - è già saldamente affermato come binomio stereotipico all'inizio del II secolo a.C., cioè si è già diffuso dal linguaggio giuridico a quello letterario (cui ancora una volta ci volgiamo, per completare la lacunosa documentazione giuridica coeva $)^{25}$.

24 Il detto aveva origine socratica: Xen. Sym. 3.4; Plat. Prot. 329e.

25 Vd. Plaut. Most. 682: bonum aequomque oras; Pers. 399; cfr. Hor. Ep. 1.12.23: verum orabit et aequum. 
In taluni casi qualifica comportamenti privati, ad esempio la determinazione di una controprestazione:

Plaut. Curc. 128-130: alias me poscit pro illa triginta minas, / alias talentum magnum: neque quicquam queo / aequi bonique ab eo impetrare («a volte mi chiede per lei trenta mine, altre volte un grande talento, e non riesco ad ottenere niente di equo e buono da lui»).

Oltre che alla comunicazione fra privati, la pertinenza del sintagma anche al linguaggio della giurisdizione è chiara da Ter. Phorm. 450: quod te absente hic filius egit, restitui in integrum aequomst et bonum, che allude alla restitutio in integrum pretoria (su cui torneremo più avanti).

La solidità (e la frequenza) del binomio bonum et aequum è confermata dalla sua occorrenza al comparativo e al superlativo, secondo formule metricamente opportune:

Plaut. Capt. 333: optumum atque aequissumum oras optumusque bomi-

num es bomo;

Plaut. Rud. 1029: hoc optumum atque aequissumumst;

Plaut. Pseud. 389: optumum atque aequissumum oras;

Turp. Com. 56: quam legere te optimum esset atque aequissimum ${ }^{26}$.

Non è facile stabilire se, al tempo di Plauto, il binomio aequum et bonum avesse un significato distinto dal semplice aequum. Per un verso, infatti, si può pensare che il linguaggio giuridico, nel suo tecnicismo, non avrebbe accostato i due termini, se non vi avesse visto un qualche significato aggiuntivo (aequum come equilibrato, bonum come utile socialmente? $)^{27}$. D'altra parte, la prolissità formulaica dei giuristi non esclude che si tratti di termini che, se avevano avuto in principio un valore distinto, si fissarono poi in un binomio sostanzialmente endiadi$\mathrm{co}$, che non dice molto più del solo aequum, come sembrano mostrare gli esempi plautini, abbastanza intercambiabili fra loro (cfr. le occorrenze raccolte nel $\mathbb{S}$ e qui nt. 25). Su un punto, tuttavia, le fonti non lasciano dubbi: anche fra ius da una parte e bonum et aequum dall'altra non vi è contrasto, bensì solidarietà. Lo mostra già un verso come Plaut. Cas. 375: Optumum atque aequissumum istud esse iure iudico (riferito a

\footnotetext{
26 Vd. anche Plaut. Most. 1147; Epid. 725.

27 In questo senso Voci, Ars boni et aequi, 292.
} 
una decisione presa utilizzando la sorte). La lex Irnitana (cap. 69) ha fatto conoscere il contenuto del giuramento prestato dai giudici, ossia se quod aequum bonumque et maxime e re communi eius municipi esse censeat, indicaturum: è probabile che il verso plautino riecheggi questa formulazione, il che implicherebbe che fosse già allora impiegata nel giuramento giudiziale.

Su questo sfondo, assume tutta la sua importanza - per il tipo di fonte e per la sua pertinenza al processo formulare - la testimonianza, troppo spesso trascurata, fornita dal SCtum de Magnetum et Prienensium litibus, cui abbiamo già accennato. Per risolvere una controversia insorta fra le due comunità greche, il senato, cui si erano rivolte, dà istruzioni al pretore Marco Emilio Lepido (a metà del II secolo a.C.) di instaurare un processo per iniuriae basato su una formula che contiene la clausola ö $\sigma o v$

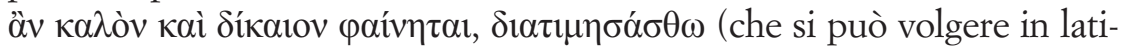
no: quantum bonum et aequum parebit, aestimanto), che è tipico della formula dell'actio iniuriarum ${ }^{28}$. Il sintagma aequim et bonum compare dunque a vari livelli, e tecnicamente, nel lessico della giurisdizione.

La convergenza fra "diritto" e aequum et bonum è attestata anche da Ter. Heaut. 642: quid cum illis agas, qui neque ius neque bonum atque aequom sciunt? («che ci fai con quelli, che non conoscono né il diritto né l'equo e il buono?»). Proprio mentre li distingue, è chiaro che per Terenzio ius e bonum et aequum sono sentiti come valori coerenti, per descrivere un uomo virtuoso.

Analogo, ma ancor più eloquente, grazie alla maggiore articolazione del contesto, Plaut. Maen. 578-583: qui neque leges neque aequom bonum usquam colunt, / sollicitos patronos babent: / datum denegant, quod datumst, litium pleni, rapaces / viri fraudulenti / qui aut faenore aut periuriis /

${ }^{28}$ Ep. M. Aemilii et SCtum de Magnetum et Prienensium litibus, lin. 57-60 Sherk:

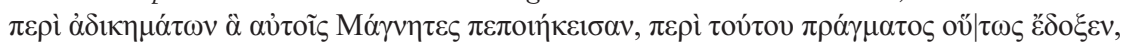

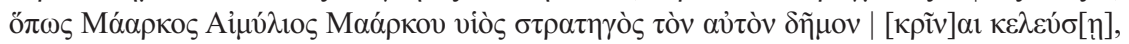

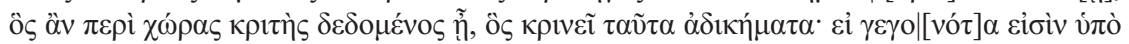

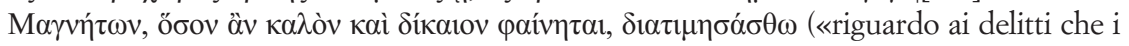
Magneti hanno commesso ai loro danni, a questo proposito così è parso al senato: che il pretore Marco Emilio figlio di Marco ordini di giudicare al medesimo popolo, che è stato dato come giudice riguardo alla controversia sul territorio, il quale giudicherà questi delitti: se sono stati commessi dai Magneti, li stimi secondo quanto sembrerà buono e equo»).

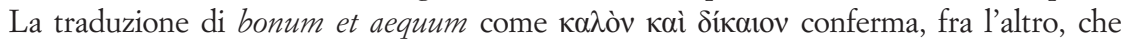

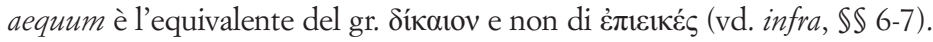


babent rem paratam (...) («quelli che non rispettano mai né le leggi né l'equo e buono, hanno avvocati solerti: negano di avere ricevuto quel che è stato loro dato, pieni di liti, rapaci, personaggi fraudolenti che si sono arricchiti o prestando a interesse o spergiurando»). Che il contesto non istauri un antagonismo fra leges (intese come diritto positivo) e aequom bonumque lo dimostra il fatto che all'opposto di quest'insieme Plauto colloca chi fa uso di patroni scaltri: gli avvocati non sono presentati dal commediografo come cultori dell'equità contro lo stretto diritto, bensì come eversori della giustizia rappresentata congiuntamente da leges e aequom bonum $^{30}$. Vale la pena anche di notare che il verbo colere anticipa la metafora dei «sacerdoti della giustizia» adottata da Ulpiano (D. 1.1.1).

Come accennato, non mi sento perciò di condividere l'interpretazione secondo la quale nel II secolo l'accostamento fra ius e (bonum et) aequum indicherebbe la scissione fra due sfere giuridiche, e sarebbe il segno di un momento nel quale il diritto, con il suo formalismo arcaico, entra in tensione con l'aequum, di cui il pretore sarebbe il solo propugnatore ${ }^{31}$.

Quest'interpretazione non persuade né per quanto riguarda il momento dell'origine (perché come s'è visto poeti e commediografi del II secolo accostano e non contrappongono le due sfere, in una dialettica che vede invece il ius coincidere con l'aequum o almeno tendere verso di esso) e neppure per quanto riguarda gli sviluppi successivi. La tensione fra ius e aequum (nel senso ora visto) non caratterizzò, infatti, solo il III-II secolo, ma l'intero diritto classico, perché i due ambiti non si saldarono mai del

29 Di analogo contenuto, ma giocato sul concetto di iustum, con un ritorno finale su aequum e ius, è Plaut. Amph.33-37: ss.: Iustam rem et facilem esse oratam a vobis volo: / nam iuste ab iustis iustus sum orator datus: / nam iniusta ab iustis impetrari non decet, / iusta autem ab iniustis petere insipientiast: / quippe illi iniqui ius ignorant neque tenent («vengo a chiedervi una cosa giusta e facile: infatti sono stato dato giustamente come oratore giusto da giusti; sarebbe indecoroso che cose ingiuste fossero chieste a giusti, e chiedere le cose giuste agli ingiusti è sciocco: infatti quelli, iniqui, ignorano e non rispettano il ius»).

30 Ovviamente una lex (in senso stretto o metaforicamente) può essere iniqua: Plaut. Merc. 817-818: ecastor lege dura vivont mulieres / multoque iniquiore miserae quam uiri (di sicuro le donne vivono secondo una dura legge, e molto più iniqua, poverette, dei mariti).

31 È questo un punto in cui non posso condividere l'analisi di Voci, Ars boni et aequi, 294 e di A. Schiavone, Ius, L'invenzione del diritto in Occidente, Torino, 2005, 115-133. Vi è certo una distinzione, e perciò a volte anche una contrapposizione, ma sottolineando la funzione critica svolta dall'aequum rispetto al ius si rischia di perdere di vista i casi normali, in cui il ius era considerato coerente con l'aequum (casi che per ciò stesso non davano motivo di invocare e citare l'aequum). 
tutto: basti pensare al rapporto fra l'intentio in ius concepta e l'exceptio pretoria che la blocca. Per evitare equivoci vale la pena di ripetere: ius e aequum sono referenti (etimologicamente e ideologicamente) distinti, che tendono a convergere, ma non sempre vi riescono. Tuttavia, è del tutto ingiustificata l'interpretazione secondo cui l'accostamento fra ius e aequum nelle fonti repubblicane indicherebbe di per se stesso una contrapposizione. La parola definitiva in proposito, mi sembra, l'ha detta Plauto (Amph. 37): iniqui ius ignorant neque tenent. (testo completo supra, nt. 29).

Non serve, né sarebbe possibile, svolgere in modo più approfondito l'argomento. Mi limito a un esempio che indica in che modo, nel corso del II secolo (se non prima), si svolgesse la dialettica fra ius e aequum (et bonum). È un passo ben noto di Ter. Phorm. 449-452: ego quae in rem tuam sint ea velim facias; mibi / sic hoc videtur: quod te absente bic filius / egit, restitui in integrum aequomst et bonum: / et id inpetrabis. Dixi («io vorrei che tu facessi quel che è più conveniente per te. Il mio parere è questo: gli atti compiuti in tua assenza da questo tuo figlio è equo e buono che siano rimessi in pristino. Ciò potrai ottenerlo. Mi sono pronunciato»). È il parere reso da un personaggio consultato, insieme ad altri, da un amico, e allude a una restitutio in integrum propter absentiam. Il passo, come ben visto dalla critica letteraria, evoca linguaggio e prassi giuridica romana, una sorta di consultazione fra giuristi ${ }^{32}$. Il senso del primo parere è accentuato dal parere di uno degli altri amici chiamati a deliberare, che si oppone alla rescissione di quel che è stato compiuto secondo le leggi: Mibi non videtur, quod sit factum legibus / rescindi posse. Sarebbe fuori luogo leggere in queste due posizioni un'evoluzione storica, il segno di un contrasto fra un imperante ius strictum e un'aequitas che stenta ancora ad affermarsi3. È invece una classica contrapposizione forense, fra due avvocati (anzi, fra due giuristi che consigliano gli avvocati) che sostengono le due posizioni in causa (e l'effetto comico è aumentato dal fatto che il terzo 'consulente' interpellato chiede maggior tempo per deliberare, una sorta di non liquet,

32 S. Papaioannou, Terence's Stock Characters and Plots: Stereotypes 'Interpreted', in S. Papaioannou (ed.), Terence and Interpretation, Newcastle upon Tyne, 2014, 159. Non manca un'allusione al ruolo del pretore.

33 Il paradosso di una simile interpretazione sarebbe di non vedere che il parere del primo "consigliere" presuppone l'esistenza, nella prassi giurisdizionale, della restitutio in integrum. Dunque, il dibattito fra aequum e lex (o ius) doveva essere già quotidianamente affrontato da giuristi e patroni nel Foro, nella dialettica fra attore e convenuto. 
al che il personaggio che aveva chiesto consiglio conclude sconsolato: Incertior sum multo quam dudum). Già a metà del II secolo, dunque, il pretore concedeva la restitutio in integrum, di essa discutevano i patroni (e i giuristi che li consigliavano) e quest'operazione era messa sotto il segno dell'aequum et bonum ${ }^{34}$.

\section{VALORI DELL'EQUITÀ IN EPOCA PRECICERONIANA}

Individuare la presenza dell'aequum (e del bonum et aequum) nel linguaggio del diritto e della giurisdizione nel III-II secolo a.C. e avere accertato che non esprime un elemento contrapposto, bensì convergente (almeno di massima) con il ius, non implica ancora averne determinato il significato. Alla luce degli esempi finora fatti, possiamo dire che senz' altro è ritenuto aequum concedere un rimedio che viene ordinariamente accordato al pubblico (dunque, aequum incorpora innanzitutto l'idea di parità di trattamento). E un tema ben sviluppato da Ter. Adelph. 800-804: [Demea:] quor emis amicam, Micio? Numqui minus / mibi idem ius tecum esse aequomst quod mecumst tibi? / Quando ego tuom non curo, ne cura meum. / [Micio:] non aequom dici' [Demea:] Non? [Micio:] nam vetus verbum hoc quidemst, / communia esse amicorum inter se omnia («[Demea:] perché compri la mia amante, Micione? Forse è equo che io abbia meno diritti verso di te di quanto tu ne hai verso di me? Dal momento che io non mi occupo del tuo, tu non occuparti del mio. [Micione:] Dici una cosa non equa. [Demea:] No? [Micione:]: Infatti c'è un vecchio proverbio che dice che tutte le cose devono essere comuni fra gli amici»).

Lo si ritroverà quattro secoli più tardi in Ulpiano $(3 \mathrm{Ad}$ ed. D. 2.2.1 pr.), a proposito dell'edictum Quod quisque iuris in alterum statuerit, ut ipse eodem iure utatur, che imponeva al magistrato giusdicente di accettare di applicare a se stesso le regole applicate agli altri: hoc edictum summam babet aequitatem, et sine cuiusquam indignatione iusta: quis enim aspernabitur idem ius sibi dici, quod ipse aliis dixit vel dici effe-

34 Potremmo assumere come rapido segno di come l'aequum entri in gioco nel soppesare le ragioni contrapposte il verso di Accio (Neoptol. fr. XII Ribbeck): Ubi nil contra rationem aequam habuit, adsensit silens ( $\ll$ Non avendo un equo argomento in contrario, assentì in silenzio»). 
cit? («quest'editto ha una somma equità, e nessuno potrebbe giustificatamente indignarsene: chi infatti si potrebbe opporre a che venga a lui applicato lo stesso ius che egli stesso ha applicato o ha fatto applicare ad altri?»).

Il testo di Terenzio introduce però anche un'altra dimensione dell'aequum, ossia che non è considerato equo (da Demea) chi si intromette in ciò che è altrui; è una sottile, ma non meno percepibile allusione al suum cuique tribuere (di cui vedremo più avanti altri notevoli esempi, da Plauto a Catone: infra, pp. 39-41).

Aequum designa inoltre l'equilibrio nel calcolo del corrispettivo, come è espresso nel fantasioso contratto (documentato per iscritto: syngraphum) di "esclusiva" per un anno dei favori di una schiava prostituta di cui parla Plauto (Asin. 229-31): [Diabolus]: (...) dic, quid me aequom censes pro illa tibi dare, I annum bunc ne cum quiquam alio sit? I [Cleareta]: Tune? Viginti minas. / Atque ea lege: si alius ad me prius attulerit, tu vale [D.]: («Di', quanto reputi equo che io ti dia per quella, perché quest'anno non stia con nessun altro? [C.]: Tu? Venti mine. E a questa condizione, che se un altro me le porta prima, ti saluto»).

Lo stesso significato di equivalenza pecuniaria ricorre in un formulario di contratto catoniano, che impone l'indennizzo del valore di mercato nel caso di mancata restituzione dell'attrezzatura (Cat. Agr. 146.3): vasa torcula, funes scalas trapetos, si quid et aliut datum erit, salva recte reddito nisi quae vetustate fracta erunt; si non reddet, aequom solvito («le attrezzature da torchio, le funi, le scale, i frantoi, e qualunque altro utensile gli sarà stato fornito, tutti correttamente li restituisca integri, tranne quelli che si saranno rotti perché vecchi; se non li renderà, paghi l'equivalente»).

Vi sono ancora un paio di passi terenziani, dunque verso la metà del II secolo a.C., in cui l'aequum prende la forma di una massima di decisione. Per la sua importanza si staglia Ter. Hecyr. 840: multa ex quo fuerint commoda, eius incommoda aequomst ferre (la meretrice Bacchis si riferisce al fatto che, dopo avere goduto dei benefici della relazione, deve accettare il matrimonio dell'amante). Il principio del nesso fra vantaggi e svantaggi si trova ripetuto in testi giuridici più tardi, ad esempio in tema di restituzione della $\operatorname{dote}^{35}$ (Ulp. 31 Ad Sab. D. 23.3.7

35 Vd. ad es. Gai. 7 Ad ed. prov. D. 10.2.19; Paul. 5 Ad Sab. D. 18.6.7 pr.; Ulp. 73 Ad ed. D. 20.1.21.2; Ulp. 39 Ad ed. D. 37.1.1-3 pr.; Gai. 14 Ad ed. prov. D. 37.1.11. 
pr.): Dotis fructum ${ }^{36}$ ad maritum pertinere debere aequitas suggerit: cum enim ipse onera matrimonii subeat, aequum est eum etiam fructus percipere («L'equità suggerisce che i frutti della dote debbano spettare al marito; infatti, poiché egli sostiene i pesi del matrimonio, è equo che egli percepisca anche i frutti») ${ }^{37}$.

Nel nesso fra vantaggio e svantaggio s'esprime un principio più profondo, ossia che in una relazione nessuno deve avvantaggiarsi a detrimento dell'altro. Tocchiamo dunque i principi fondamentali della teoria della giustizia, alterum non laedere, summ cuique tribuere (cfr. Cic. Off. 1.20; Ulp. 1 Reg. D. 1.1.10), quegli stessi che - incidentalmente avevamo visti ricordati da Adam Smith a proposito della equa retribuzione degli operai.

La qualificazione aequum (o iniquum) est si trova anche a proposito del rapporto fra colpa e punizione o offesa e beneficio, come in Ter. Hecyr. 740: nunc si id facis facturave es, bonas quod par est facere, I inscitum offerre iniuriam tibi me inmerenti iniquomst (detto a una meretrice che rivendica la propria correttezza, al di là della condizione sociale: «se ora fai o farai quel che è adeguato che facciano le donne perbene, sarebbe iniquo che io senza pensarci troppo facessi un torto a te che non lo meriti»).

È particolarmente significativo l'uso di questo criterio di valutazione nella Pro Rhodiensibus di Catone, che invita i senatori a riflettere sull'iniquità di punire chi abbia solo avuto l'intenzione di fare del male ad altri, senza passare all'atto (Cato Orig. 5.4): ecquis est tandem qui vestrorum, quod ad sese attineat, aequum censeat, poenas dare ob eam rem quod arguatur male facere voluisse? («c'è forse qualcuno di voi che, per quanto lo riguarda, riterrebbe equo essere punito perché gli si imputa di avere avuto l'intenzione di agire male?»).

Più lontano da questa idea di parità è il caso già visto (supra, p. 3031) della restitutio in integrum, in cui si esprime l'idea che si debba tutelare chi, senza sua colpa, si sia trovato assente. Qui l'aequum et bonum assume evidentemente un significato che oltrepassa quello della semplice eguaglianza; mantiene tuttavia un legame con questo nocciolo semantico, ossia di assicurare un processo imparziale e di evitare un vantaggio ingiu-

\footnotetext{
36 Vd. supra, nt. 1.

37 Importante Stagl, Die Ausgleichung von Vorteil und Nachteil als Inhalt klassischer aequitas, 698-700.
} 
stificato $^{38}$. Mi pare che quest'applicazione sia in linea con quanto si legge in uno dei primi documenti che presentano una riflessione esplicita sul rapporto fra ius e aequum et bonum, ossia la Rhetorica ad Herennium, all'inizio del I sec. a.C. (2.20): ex aequo et bono ius constat quod ad veritatem <et utilitatem> communem videtur pertinere; quod genus, ut maior annis LX et cui morbus causa est cognitorem det. Ex eo vel novum ius constitui convenit ex tempore et ex hominis dignitate («si fonda sul bonum et aequum il ius che si considera conforme alla verità e all'utilità comune; è di questo genere la regola che (il pretore) dia un rappresentante processuale a chi ha più di sessant'anni e a chi ha un impedimento di salute. In base ad esso è conveniente anche costituire un ius nuovo in considerazione delle circostanze e della posizione della persona»). Nella prima proposizione si riconduce al bonum et aequum la regola (sancita dal pretore, con un apposito editto applicabile a tutti), che per supplire a situazioni di debolezza che renderebbero dispari il processo, il magistrato deve fornire un cognitor, che assista la parte debole per età o malattia. Come si vede, non è distante la ratio che ispira uno dei consulenti raffigurati da Terenzio a proporre la restitutio in integrum a chi non aveva potuto difendersi a motivo dell'assenza incolpevole. Colpisce che in entrambi i casi - in Terenzio e nella Rhetorica ad Herennium - il referente sia specificamente il bonum et aequum, quasi che il riferimento al bonum, all'utilità sociale, possa corroborare in questo caso l'equità pura e semplice ${ }^{39}$.

\section{L'AEQUITAS E LA VIRTÙ GRECA DELLA GIUSTIZIA}

Su questo fondo antico e romano della nozione di aequum si sovrappone, in un momento che è difficile stabilire, ma che come vedremo si può riportare almeno al II secolo a.C., il concetto greco di

38 In un altro esempio, viene ritenuto aequum che, avvenuta l'agnizione del nipote, lo zio paterno che aveva ricevuto in eredità i beni del fratello, li restituisca al figlio legittimo del defunto: Plaut. Poen. 1080-81: Paterna oportet filio reddi bona: / aequomst habere bunc bona quae possedit pater.

39 Nella seconda proposizione di Rhet. Her. 2.20, il retore osserva che in taluni casi, legati alle circostanze specifiche, si può statuire un ius fino ad allora non previsto (novum). La contrapposizione è appunto fra la regola espressa in generale nell'editto e quella che si può statuire con un decreto, causa cognita. In entrambi i casi, è bene osservarlo, il bonum et aequum non si oppone al ius, bensì ne è il fondamento. 


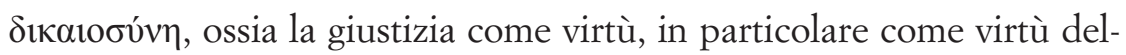
l'attribuzione o retribuzione proporzionata.

Il concetto è specialmente noto attraverso Aristotele, ma era già espresso (e criticato) da Platone, che lo attribuiva a Simonide, il poeta di Ceo vissuto fra VI e V secolo («Pensava che la giustizia fosse dare a ciascuno ciò che gli compete, e ciò che gli compete chiamò invece ciò che è dovuto» $)^{40}$; trovò poi ampia accoglienza nello stoicismo ${ }^{41}$. In breve, secondo Aristotele (De Virtutibus et Vitiis, 1250b) il proprio

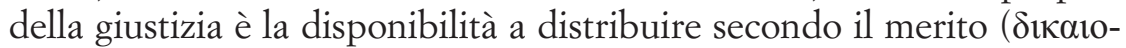

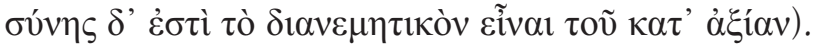

Per meglio apprezzare la ricezione latina di questa nozione, rammentiamo come Aristotele pervenga alla definizione della giustiza distributiva e della giustizia correttiva, rifacendoci alla canonica argomentazione svolta nel libro V dell'Etica Nicomachea e alla sua sintesi nei Magna

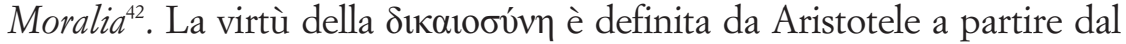
suo opposto, il vizio della $\pi \lambda \varepsilon o v \varepsilon \xi i ́ \alpha$, dell'avidità, di cui pecca chi si avvantaggia più del dovuto. La giustizia concepita come virtù in senso specifico (in senso generale essa si identifica invece con il rispetto della legge) consiste nella medietà tra due quantità eccessive, fra il troppo e il troppo poco. Ne consegue, come un'evidenza, che se l'ingiusto è il più (il

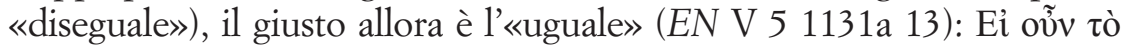

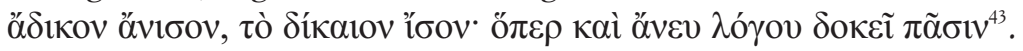

La giustizia $(\delta ı \alpha \iota เ \sigma u ́ v \eta)$ così definita si articola in due tipi, diversi sia riguardo alla natura dei rapporti sociali coinvolti, sia riguardo al

40 Plato Rep. I 332c (trad. it. G. Gabrieli). Vd. A. Jellamo, Il cammino di Dike. L'idea di giustizia da Omero a Eschilo, Roma, 2005, VII. Quest'idea circola già nel concetto pitagorico di proporzionalità.

41 Zeno SVF I fr. 200; Chrysipp. SVF III fr. 255; 264; 266.

${ }^{42}$ Nella bibliografia immensa, vd. J. Brunschwig, Rule and exception: on the Aristotelian theory of equity, in M. Frede - G. Striker (eds.), Rationality in Greek Thought, Oxford, 1996, 115-155; più recentemente, buona analisi in S. Contreras, La justicia en Aristóteles. Una revisión de las idéas fundamentales de Ethica Nicomachea, in Ágora. Estudos Clássicos em Debate 14 (2012) 63-80; attenti anche al collegamento con la teoria contemporanea del diritto i saggi raccolti in L. Huppes-Cluysenaer - N.M.M.S. Coelho (eds.), Aristotle and the philosophy of law: theory, practice and justice, Dordrecht, 2013; vd. il saggio di M. Vegetti.

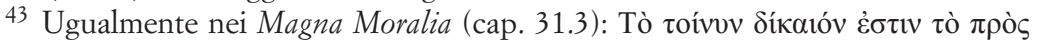

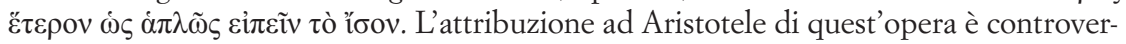
sa: sicura è la fedeltà al suo pensiero. 
calcolo del rapporto di uguaglianza. La giustizia del primo tipo «è quella che si attua nella distribuzione di onori, di denaro o di quant'altro si può ripartire tra i membri della cittadinanza (giacché in queste cose uno può avere una parte sia disuguale sia uguale a quella di un altro), l'altra è quella che apporta correzioni nei rapporti privati» (EN V 5 1130b 3033). La giustizia del primo tipo (distributiva) opera in base alla proporzione (fra i meriti e i beni attribuiti a ciascuno), la seconda (correttiva o commutativa o retributiva) in base all'aritmetica: «il giudice ristabilisce l'uguaglianza, cioè, come se si trattasse di una linea divisa in parti disuguali, egli sottrae ciò di cui la parte maggiore sorpassa la metà e l'aggiunge alla parte minore» (EN V 5 1132a 25-28).

Non sappiamo quando e chi abbia trasposto in latino le riflessioni

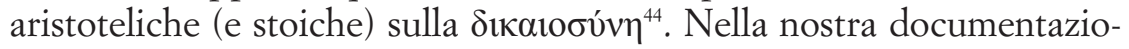
ne, la definizione greca di giustizia come uguaglianza (in senso distributivo) appare tradotta in latino nei primi anni del I secolo a.C., in due opuscoli di retorica giudiziale (cioè con intenti pratici e, per così dire, di seconda mano, senza ambizione filosofica).

I due testi sono pressoché contemporanei, e forse riflesso dell'insegnamento di uno stesso maestro di retorica, l'uno di autore ignoto, l'altro del giovane Cicerone:

Her. (3.3): Iustitia est aequitas ius uni cuique rei tribuens pro dignitate cuinsque

(«la giustizia è l'equità che attribuisce a ciascuna cosa il ius in rapporto alla dignità di ciascuno»).

Cic. Inv. 2.160: Iustitia est habitus animi communi utilitate conservata suam cuique tribuens dignitatem

(«La giustizia è l'atteggiamento mentale che attribuisce a ciascuno la propria dignitas, preservando l'utilità comune»).

Definizione analoga si ritrova in età severiana in Ulpiano $(1 \mathrm{Reg}$. D. 1.1.10 pr.): Iustitia est constans et perpetua voluntas ius suum cuique tribuend $i^{45}$ e allusioni si trovano sparse nella letteratura latina ${ }^{46}$.

${ }^{44}$ G. Thome, Iustitia. Geschichte eines Wortes und einer Idee, in Anregung. Zeitschrift für Gymnasialpädagogik 45 (1999) 150-168.

45 Sulla definizione di giustizia ulpianea, vd. per tutti Waldstein, Ist das suum cuique eine Leerformel?, 169 ss.; G. Falcone, Ius sum cuique tribuere, in Annali dell'Università di Palermo 52 (2010) 135-176 (qui p. 136 n. 3 altra bibliografia); Finkenauer, Iustitia und iustus, 287-300, spec. 288-289. Vd. anche infra $\$ 10$.

46 Ad es. Cic. Brut. 85: erat omnino tum mos ... ut faciles essent in sum cuique 
Ai nostri fini, è sufficiente notare, confrontando le due definizioni parallele più antiche, che entrambe adottano come definiendum (equiva-

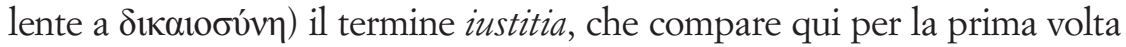
(e deve essere considerato una formazione colta, probabilmente escogitata proprio per tradurre il nome greco $)^{47}$. La Rhetorica ad Herennium adotta come oggetto della distribuzione il ius e come misura la dignitas, mentre Cicerone incorpora nella dignitas l'oggetto e la misura. In Cicerone si accenna alla conservazione della utilitas communis, cioè alla funzione sociale della iustitia. In entrambi i contesti, infine, la iustitia è una virtù, dunque un atteggiamento soggettivo. Ciò è detto esplicitamente nella definizione ciceroniana ${ }^{48}$ (cui si avvicina in questo punto quella ulpianea) e si ricava senz'alcun dubbio dal contesto in cui è inserita la definizione della Rhetorica ad Herennium, che è appunto inclusa in un elenco di virtutes. Spicca nella definizione della Rhetorica il termine aequitas. È acuta l'interpretazione secondo cui aequitas qui corrisponde in qualche modo a babitus animi, indica cioè anche nella Rhetorica ad Herennium una disposizione d'animo (ossia è anch'essa un riferimento esplicito alla virtù); a differenza dell'espressione anodina usata da Cicerone, aequitas include tuttavia un'allusione al contenuto di questa disposizione d'animo, cioè l'equanimità, l'equilibrio ${ }^{49}$. Anche se in forma indiretta, dunque, già all'inizio del I secolo a.C. l'idea di "eguaglianza" si fa strada nella trasposizione della

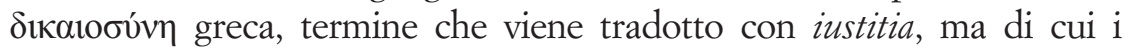
Romani avvertono l'affinità con l'aequum ${ }^{50}$. Considerato infatti il modo in

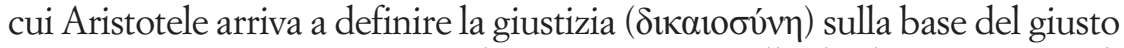

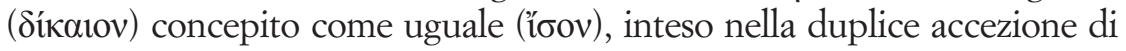

tribuendo; Fin. 3.67; 5.65; Leg. 1.19 (vd. infra); Off. 1.15; Rutil. Lup. Schem. 1.10; Apul. Apol. 1.16; Tryph. 9 Disp. D. 16.3.31.1: Et probo banc esse iustitiam, quae suum cuique ita tribuit, ut non distrabatur ab ullius personae iustiore repetitione; Amm. 22.9.9; 22.10.1; Zeno Tract. 2.6.9.

47 Così giustamente Thome, Iustitia, 152, 156-157; Ostwaldt, Aequitas und Justitia, 72.

${ }^{48} \mathrm{Nel}$ De inventione, la definizione di iustitia si iscrive nella definizione delle quattro virtù, che a loro volta servono a descrivere l'bonestum, che è il fine delle orazioni epidittiche e (con l'utile) delle deliberative. L'aequitas è invece il fine delle orazioni giudiziarie e considerata aspetto facente parte dell'bonestas (Inv. 2.156).

49 Ostwaldt, Aequitas und Justitia, 73-74.

50 Una connessione fra aequitas e giustizia in senso distributivo e retributivo (anche se in un contesto diverso) si trova in Cic. Top. 9: ius civile est aequitas constituta eis, qui eiusdem civitatis sunt, ad res suas obtinendas. 
punto di riequilibrio fra due interessi in conflitto (giustizia correttiva) oppure come corrispondenza fra merito e risorsa allocata (giustizia distributiva), si vede bene perché aequitas sia stato individuato come termine

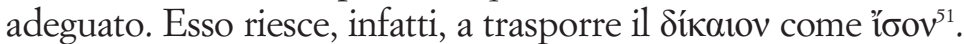

$\grave{E}$ quanto afferma in modo estremamente condensato un brano ben noto di Cicerone, che accosta il significato di lex e di vónos, e le rispettive (par)etimologie (Leg. 1.19): itaque arbitrantur (scil.: doctissimi viri) prudentiam esse legem, cuius ea vis sit, ut recte facere iubeat, vetet delinquere, eamque rem illi Graeco putant nomine a suum cuique tribuendo appellatam, ego nostro a legendo; nam ut illi aequitatis, sic nos dilectus vim in lege ponimus, et proprium tamen utrumque legis est $t^{52}$. Il brano si può rendere come segue: «Pertanto i filosofi ritengono che il discernimento sia legge, la cui essenza consiste nell'ordinare di agire correttamente e vietare di delinquere, ed essa costoro in base al termine greco $(=v$ ó $\mu \mathrm{s})$ ritengono che sia stata chiamata dal distribuire a ciascuno il suo (= vé $\mu \varepsilon ı v$, distribuire), io in base al termine latino (=lex) da scegliere (= legere). Infatti, come i Greci considerano che l'essenza della legge stia nell'uguaglianza (aequitas), così noi la riponiamo nella scelta, e tuttavia entrambi gli aspetti sono propri della legge».

Attraverso il parallelismo, Cicerone enuclea due aspetti della legge: dal termine latino lex, la funzione del legere, scegliere fra bene e

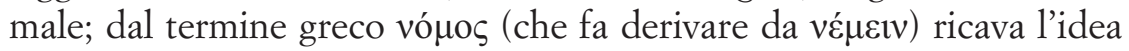

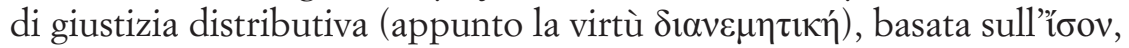
l'uguaglianza, ed è quest'ultimo valore che Cicerone traduce precisamente con aequitas.

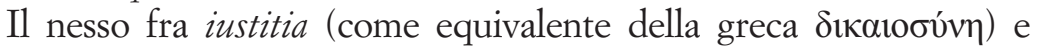
aequitas resta peraltro problematico, nel senso che a volte i due termini sembrano usati sinonimicamente o come un'endiadir3 ${ }^{53}$, altre volte le nozio-

51 Significativo anche Apul. Plat. Dogm. 2.7: duabus autem aequalibus de causis utilitatem hominum iustitia regit, quarum est prima numerorum observantia et divisionum aequalitas et eorum quae pacta sunt symbole, ad haec ponderum mensurarumque custos et communicatio opum publicarum; secunda finalis est et veniens ex aequitate partitio, ut singulis in agros dominatus congruens deferatur ac servetur, bonis optima portio, minor non bonis; ad hoc bonus quisque natura et industria in bonoribus et officiis praeferatur, pessimi cives luce careant dignitatis.

52 Su questo brano vd. Falcone, Ius suum cuique tribuere, 149 nt. 30.

53 Es. Cic. Top. 90 (se non è una glossa); Amic. 82. 
ni vengono considerate affini, ma distinte ${ }^{54}$. Senza potere approfondire la

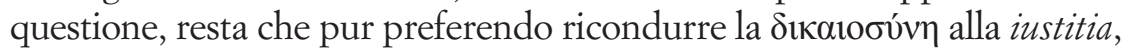
per Cicerone anche l'aequitas vi aveva un legame.

Naturalmente, proprio il fatto che emerga in questo momento l'astratto (attestato per la prima volta proprio nella definizione della Rhetorica ad Herennium) è il segno che si tratta di una riflessione colta. Ma occorre fare una duplice precisazione.

La prima è che la parola Aequitas (in una forma arcaica) appare già in una coppa di argilla nera smaltata, proveniente da Vulci (ma di fattura forse romana), dal 1847 conservata al British Museum, databile al 280 a.C. La parola appare al genitivo, nella forma aecetiai (riconducibile al lemma aecetia/aequitia) e si intende perciò che designi la destinataria della coppa, ossia la proprietaria o più probabilmente la dea Aequitas ${ }^{55}$. Sarebbe affrettato considerare questo reperto come documento dell'importazione precoce della teoria della giustizia greca a Roma, perché resta da capire se la (probabile) divinità cui è dedicata concerna la giustizia o, come si riscontra più tardi nel catalogo delle virtù imperiali, evochi la correttezza dei pesi e della monetazione o infine, come appare più probabile, presieda alla correttezza degli scambi nel mercato ${ }^{56}$. Quale che sia la risposta, la testimonianza resta fondamentale, perché dà la prova che già agli inizi del III secolo a.C., due secoli prima che appaia nei manuali di retorica, i Romani avevano elaborato l'astratto aequitas; aequum non era solo un concetto operativo molto diffuso, ma aveva già subito una rielaborazione riflessiva.

Una seconda considerazione ci orienta nella stessa direzione. Oltre un secolo prima della Rethorica ad Herennium e del De inventione di Cicerone, la definizione di giustizia distributiva sottende una pagina di Plauto, scritta intorno al 205 a.C. (Mil. 725-732): aequom fuit / deos paravisse, uno exemplo ne omnes vitam viverent / sicut merci pretium statuit quist probus agoranomus: / quae probast, pro virtute ut veneat, I quae inprobast, pro mercis vitio dominum pretio pauperet: / itidem divos dispertisse vitam bumanam aequom fuit: / qui lepide ingeniatus esset, vitam ei longin-

54 Es. Cic. Off. 1.64.

55 A.H. Smith, Corpus Vasorum Antiquorum: Great Britain 1, British Museum 1, London, 1925, IV D c, Pl. 6, nr. 8 = CIL I2 439. Coppa di argilla nera smaltata, a forma semisferica; a metà della parete interna, festone vegetale circolare, dipinto in colore chiaro; sul fondo, quattro rosette a stampo. Fattura attribuita a Roma.

${ }^{56}$ Così Ostwaldt, Aequitas und Justitia, 80. 
quam darent, I qui inprobi essent et scelesti, is adimerent animam cito («sarebbe stato equo che gli dei avessero fatto in modo che non vivessero tutti secondo una stessa misura; come un probo vigile del mercato stabilisce un prezzo per la merce, quella che è buona, che sia venduta in proporzione alla sua bontà, quella che è scadente, faccia conseguire al padrone un prezzo più basso in proporzione al vizio della merce, allo stesso modo sarebbe stato equo che gli dei avessero distinto la vita degli uomini, dando una lunga vita a quello che ha uno spirito cordiale, e facendo rendere presto l'anima a quelli che sono improbi e malvagi»).

Naturalmente, si tratta di un'eco letteraria, che riprende il modello della commedia nuova ellenistica. Ma resta che lo scrittore romano intende perfettamente il concetto, lo diffonde e lo pone sotto il segno dell'aequum. Ancora più significativo è che lo ambienti nel mercato, segno che aequitas era sentita innanzitutto come corrispondenza patrimoniale negli scambi.

Oltre a questa plautina, ne avevamo già incontrato un'allusione in

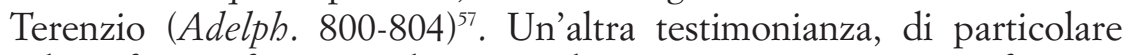
valore, finora sfuggita, è di Catone il Censore, in un'orazione riferita in parte da Gellio e di cui è andato perduto il titolo, forse De sumptu suo, e apparentemente posteriore al settantesimo anno, cioè al 164 (fr. 218a C. - S.C. $=\mathrm{ORF}^{3}$ 174: Gell. 13.24.1): Neque mibi - inquit (scil.: Cato) aedificatio neque vasum neque vestimentum ullum est manupretiosum neque pretiosus servus neque ancilla. Si quid est - inquit - quod utar, utor; si non est, egeo. Su<u>m cuique per me uti atque frui licet («Non ho alcun fabbricato né suppellettile né vestito impreziosito dalla sartoria né alcuno schiavo o ancella di gran prezzo. Se ho qualcosa da usare,

57 Vd. supra, p. 31-32. Altra allusione plautina nel Rudens (1230-31): Aequum videtur tibi, ut ego, alienum quod est, / meum esse dicam? («Ti pare equo che io affermi che è mio quello che è altrui?»). Il tessuto finemente giuridico della commedia è stato da poco messo in luce, vd. L. Pellecchi, Per una lettura giuridica della Rudens di Plauto, in Athenaeum 101 (2013) 103-162. Cfr. anche Rud. 710: Iniqua bace patior cum pretio tuo (...) ius meum ereptumst mibi. / Eas mihi ancillas invito me eripis («Queste iniquità, me le pagherai! Mi è stato sottratto un diritto mio, mi sottrai contro la mia volontà quelle ancelle»). Vd. anche, sempre nel II sec., Atilius (Comoed. frag.): I Suam cuique sponsam, mibi meam: suum cuique amorem, mibi meum. Si consideri infine l'orazione di Q. Cecilio Metello (cens. 131 a.C.) riferita in Gell. 1.6.7: Is demum deos propitios esse aecum est, qui sibi adversarii non sunt («E equo che gli dei siano propizi soltanto a quelli che non sono loro avversari»). 
la uso, se non l'ho, ne faccio a meno. Per quanto sta a me, a ciascuno è lecito usare e fruire solo del suo». Poi aggiunge: «Mi rimproverano che mi mancano molte cose, io rimprovero a loro di non sopportarne la mancanza»). Catone esprime la sua frugalità, con un argomento in due tempi: innanzitutto, dichiara di non possedere beni di lusso, ma solo funzionali. Poi, che la sua regola di vita è di accontentarsi: usa quel che ha, altrimenti vi rinuncia volentieri. Di conseguenza, si tiene al proprio e s'astiene da qualsiasi appetito, dal desiderio di appropriarsi dei beni altrui: $s u<u>m$ cuique per me uti atque frui licet esprime bene la giustizia come misura, a differenza di chi non sa trattenere la propria avidità. La costruzione di utor con l'accusativo rende ancora più scoperto il calco del suum cuique tribuere ${ }^{58}$. Con il che si viene a confermare che fra III e II secolo sono già tracciate le linee di pensiero che emergeranno in forma più esplicita in età ciceroniana.

\section{L'AEQUITAS NON È L'EPIEIKEIA}

Prima di trarre le conseguenze di questo tragitto concettuale, occorre affrontare un equivoco tanto diffuso quanto apparentemente inestirpabile, che costituisce il principale inciampo nella comprensione dell'aequitas romana, ossia l'erronea equivalenza che viene spesso isti-

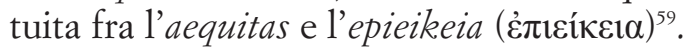

58 Un impiego della giustizia in senso distributivo è in Cato, fr. 223 C.-S.C. $\left(\mathrm{ORF}^{3}\right.$ 252: Fest. 408, 31 L.): lege libertate re publica communiter uti oportet; gloria atque bonore, quomodo sibi quisque struxit («Bisogna fruire in modo comune a tutti del diritto, della legge, della libertà, della cosa pubblica; della gloria e dell'onore, nella misura in cui uno se li è conquistati»).

59 Contestava già l'identificazione l'importante studio di Biscardi, On aequitas and epieikeia, 1-11; sinteticamente R. Gröscher - A. Wiehart, Die Römer und die Kunst der Jurisprudenz, in R. Gröscher - C. Dierksmeier - M. Henkel - A. Wiehart, Rechts- und Staatsphilosophie. Ein dogmenphilosophischer Dialog, Berlin - Heidelberg - New York, 2000, 73; eccellente anche su questo punto l'analisi di Ostwald, Aequitas und Justitia, 39-42. Identificano invece ancora epieikeia e aequitas, fra i recenti, ad es. Th. Ziolkowski, The mirror of justice. Literary reflections of legal crises, Princeton, NJ, 1997, 165-167, particolarmente istruttivo perché mostra, nel ripercorrere in modo erudito il dibattito dalle fonti romane all'Umanesimo giuridico, che questa identificazione è appunto frutto della rilettura medievale e moderna - attraverso l'aristotelismo - del 
Va premesso che, nonostante l'assonanza, equità e epieikeia (ossia

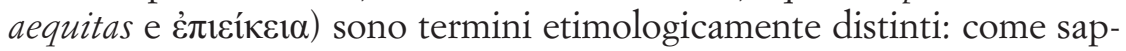
piamo, aequitas (tramite aequus) viene da aeq- radice che significa

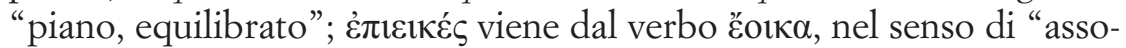

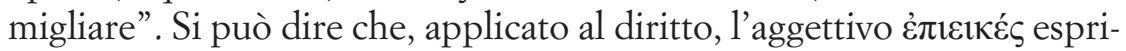
me ciò che è "conveniente" in quanto "adeguato", cioè in ultima analisi "somigliante" alla situazione che vuole regolare ${ }^{60}$.

Mi rifaccio brevemente ancora ad Aristotele: la discussione più

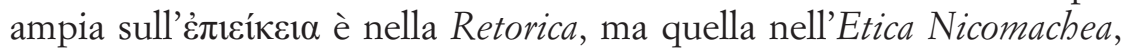
proprio per la sua organicità filosofica iscritta in una teoria della giustizia, ci indirizza in modo più nitido ${ }^{61}$. Il problema, per Aristotele, è quel-

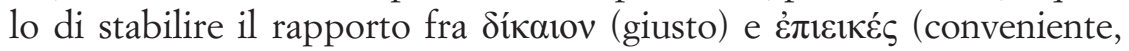
adeguato). Il fenomeno deriva dal fatto che «la legge è sempre una norma universale, mentre di alcuni casi singoli non è possibile trattare correttamente in universale $»^{62}$. «Quando, dunque, la legge parla in universale, ed in seguito avviene qualcosa che non rientra nella norma universale, allora è legittimo, laddove il legislatore ha trascurato qualcosa e non ha colto nel segno, per avere parlato in generale, correggere l'omissione, e considerare prescritto ciò che il legislatore stesso direbbe se fosse presente, e che avrebbe incluso nella legge se avesse potuto cono-

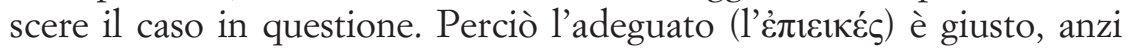

diritto romano; Solidoro Maruotti, Tra morale e diritto, 30-31. Traduce «equità» C. Mazzarelli, nella peraltro ottima ed. di Aristotele, Etica Nicomachea, Milano, 2000, 221 (che segue la num. del Didot, anche qui adottata).

${ }^{60}$ L'aggettivo (e altri con diversi prefissi) esprime «non l'idée de ressemblance, mais celle de convenance etc., avec un sens intellectuel et moral»: P. Chantraine, Dictionnaire étymologique de la langue greque. Histoire des mots, Paris, 1983, 354-355. Cfr. P. Chiron, Quelques observations sur un intraduisible célèbre (epieikes, epieikeia), in Aevum Antiquum n.s. 9 (2009) 41-49.

61 Convincente la ricostruzione dell'epieikeia correttiva proposta da F. Piazza, La virtù di Emone: riflessioni sull'epieikeia greca, in Aevuum Antiquum n.s. 9 (2009) 3. 36, seppure con le osservazioni di M. Bonazzi, La virtù di Creonte: qualche osservazione sul rapporto tra epieikeia e dike, ibid., 37-40 e di M. Pakaluk, Epieikeia in Aristotle's Nicomachean Ethics, ibid., 71-84. Importante rilettura complessiva in E. Harries, How strictly did the Athenian courts apply the law? The role of epieikeia, in Bullettin of the Institute of Classical Studies 56 (2013) 25-48, il quale giustamente nega «any reason to see in epieikeia an appeal to extra-legal considerations or an argument for ignoring the law altogether».

${ }^{62}$ EN V 10 1137b 12-14. 
migliore (...) del giusto che è approssimativo per il fatto di essere uni-

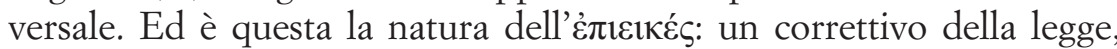
laddove è difettosa a causa della sua universalità» ${ }^{63}$. Di qui il paragone del decreto correttivo con «il regolo di piombo usato nella costruzione di Lesbo: il regolo si adatta alla configurazione della pietra e non rimane rigido, come il decreto si adatta ai fatti $\diamond^{64}$.

Quest'operazione giudiziale di correzione della legge non è affatto un'applicazione benevola. Il punto giuridico è che la direttiva per chi opera la correzione, l'adeguamento della norma generale al caso, è di «pensare come il legislatore», ossia «considerare prescritto ciò che il legislatore stesso direbbe se fosse presente, e che avrebbe incluso nella legge se avesse potuto conoscere il caso in questione». Qui si arriva al centro della questione: siccome il legislatore opera (o dovrebbe operare) secondo giustizia, ossia ricercando l'toov, l'uguaglianza, anche chi corregge la legge si deve ispirare allo stesso criterio. Rientra perciò in gioco l'equo/aequum, ma in un modo ben diverso da

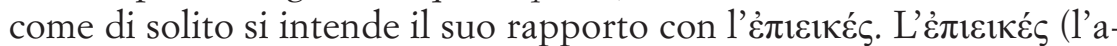
deguato, il conveniente) è il risultato di una correzione del diritto legislativo che avviene nel caso concreto adottando il criterio che ispira il diritto, ossia (almeno in linea di principio) l'equità. È come dire che l'uno è il risultato, l'altro il criterio, dunque sono ben distinti. In sintesi, l'ż $\pi 1 \varepsilon i ́ \kappa \varepsilon 1 \alpha$ non è l'equità, ma è la virtù morale che consiste nel-

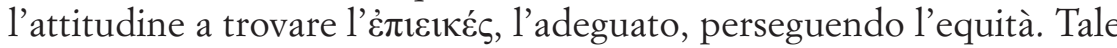
attitudine all'adeguamento è richiesta al giudice, che esamina il caso come se fosse il legislatore (dunque senza preferenza per l'una o l'altra parte), al fine di ristabilire l'uguaglianza che, per un difetto, la legge non garantisce ${ }^{65}$. Dal punto di vista diverso, di chi, per effetto della

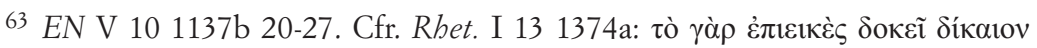

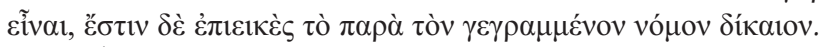

${ }^{64}$ EN V $101137 \mathrm{~b} 29-32$.

${ }^{65} \mathrm{La}$ ricerca dell'adeguamento della regola generale al caso concreto (omesso volontariamente o imprevisto dal legislatore) implica varie strategie, come privilegiare la volontà del legislatore rispetto alla lettera della legge; trattare in modo diverso i delitti colposi e dolosi; considerare non la parte, ma il tutto (vd. in particolare Arist. Rhet. I 13 1374b, su cui G. Cambiano, Come nave in tempesta. Il governo della città in Platone e Aristotele, Roma-Bari, 2016, 192-195). Benché questi orientamenti interpretativi possano sembrare "misericordiosi", essi - applicati dal giudice (vd. nt. succ.) - sono in realtà mezzi e principi che portano a rispettare l'uguaglianza (in particolare, aiutano a 
correzione, riceve meno di quanto la lettera della legge (o il suo diritto

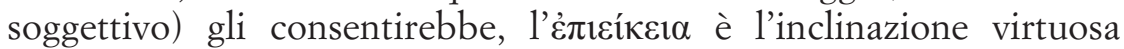
$(\dot{\eta} \tilde{\varepsilon} \xi 1 \zeta)$ a rinunciare a ciò che potrebbe pretendere (è l'uomo definito

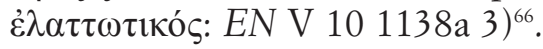

Per quanto l'errata identificazione sia radicata nella tradizione medievale e moderna ${ }^{67}$ (al punto che si potrebbe dire che ormai per molti l'equità "è" l'epieikeia, e tale resterà a dispetto di ogni evidenza), il chiarimento aiuta a acquistare consapevolezza delle fonti ${ }^{68}$. L'equivoco da evitare è di confondere l'ż $\pi ı \varepsilon i ́ k \varepsilon ı \alpha$ correttiva esercitata dal giudice con l'aequitas criterio di uguaglianza, con il rischio ulteriore

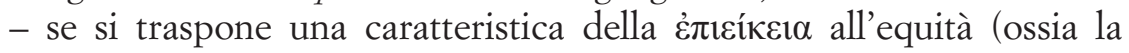
rinuncia dell'interessato a esigere tutto quello che la legge permetterebbe) - di ritenere che l'equità (del giudice) consisterebbe in un atteggiamento remissivo e misericordioso ${ }^{69}$.

rispettare la regola di trattare ugualmente casi uguali e diversamente quelli dissimili) e a correggere la norma generale che sarebbe altrimenti ingiusta nel caso specifico (ad es., se non distinguesse fra dolo e colpa).

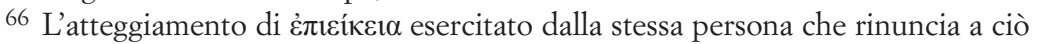
che potrebbe pretendere è opportunamente definito come "riflessivo" (o passivo) da Brunschwig, Rule and exception, 124-126: esso è giustamente considerato "esterno al diritto", nel senso che non è una correzione della regola proposta dal giudice.

67 Vd. già Thom. Summa Theol., IIa-IIae, q. 120, art. I co: Epieikeia, quae apud nos dicitur aequitas.

68 A complicare il rapporto fra le due nozioni (e a spiegare in parte la difficoltà di districarle, nonostante esse siano totalmente distinte sul piano semantico) contribui-

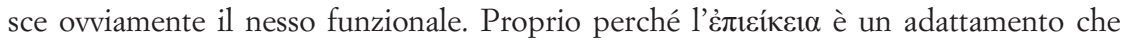
cerca di ristabilire l'eguaglianza (aequitas), alcune fonti possono usare aequitas per alludere all'operazione di adattamento al caso concreto. Mi pare molto ben espresso dal giurista di fine II sec. d.C., Quinto Cervidio Scevola (L. s. quaest. publ. tract. D. 44.3.14), a proposito della possibilità per l'avente causa di avvalersi del periodo di possesso maturato dal dante causa. I casi sono talmente vari che bisogna rinunciare a statuire una regola generale una volta per tutte (osservazione che evoca il problema aristotelico della norma che erra perché parla in universale), dovendosi risolvere il caso di volta in volta basandosi sull'aequitas (De accessionibus possessionum nibil in perpetuum neque generaliter definire possumus: consistunt enim in sola aequitate).

${ }^{69}$ La sovrapposizione mi sembra spieghi la glossa di Donat. Ad Ter. Ad. 51: ius est, quod omnia recta et inflexibilia exigit. Aequitas est, quae de iure multum remittit, spesso elevata a definizione della aequitas perché letta fuori contesto. Si tratta della nota al verso 51-52 degli Adelphoe terenziani: non necesse babeo omnia pro meo iure agere, parole con 


\section{AEQUiTAS E IUSTITIA NELL'ICONOGRAFIA}

L'indagine su un concetto non è costretta ad affidarsi solo all'etimologia o agli usi di una parola. Anche l'iconografia è vettore, spesso molto efficace, del contenuto che i contemporanei assegnano a un'idea. I giuristi romani, lo abbiamo ricordato in esordio, ritenevano che il giudice dovesse aequitatem ante oculos habere, dunque ritenevano che avesse fattezze riconoscibili ${ }^{70}$. Come la vedevano?

Nel caso dell'aequitas (e della iustitia) la ricerca iconografica ha compiuto un vero salto di qualità con la recente indagine di Lars Ostwaldt, dei cui risultati mi avvalgo ${ }^{71}$. Anche in questo caso, come nel chiarire gli usi lessicali, occorre compiere opportune distinzioni.

La rappresentazione dell'aequitas compare per la prima volta sulle monete di Galba, nel 68 d.C., con particolare eleganza nell'asse di bronzo al cui verso è rappresentata come una donna, rivolta a destra, che reca nella mano sinistra un'asta (scettro?) e nella destra una bilan$\mathrm{cia}^{72}$ (Fig. 1). La legenda Aequitas rende certa l'identificazione.

La figurazione ricompare frequentemente nella monetazione imperiale successiva, spesso contraddistinta, invece che dallo scettro, da una cornucopia: la si trova così ancora nelle monete di Diocleziano (ad es. nell'antoniniano della zecca di Lione, RIC V 12, del 292-293).

Secondo un'idea molto diffusa, l'Aequitas rappresenterebbe l'o-

cui il padre adottivo spiega che, pur potendo esercitare inflessibilmente la sua patria potestà (chiamata nel contesto anche imperium), adotta un comportamento più moderato nei confronti del figlio (ispirato esplicitamente al bonum et aequum). Non si tratta di una contrapposizione fra ius civile e aequitas (né riguarda l'atteggiamento del giudice o dell'interprete); si riferisce alla posizione di chi pur potendo esercitare una pretesa piena, ritiene più confacente rinunciarvi in parte. Il commentatore è dunque influenzato dal concetto

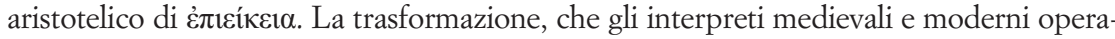
no a partire da questo testo, dell'equità (del giudice) in misericordia, è estranea al lessico dell'equità romana (cfr., a proposito di un processo criminale, Val. Max. 8.1. absol. 2: misericordia ergo illam quaestionem, non aequitas rexit).

70 Ulp. 27 Ad ed. D. 13.4.4.1.

71 Vd. nt. 7. Per la storia iconografica medievale e moderna, importante anche A. Prosperi, Giustizia bendata. Percorsi storici di un'immagine, Torino, 2008.

72 RIC I² 491 (ca. dicembre 68); Münzkabinett, Staatliche Museen zu Berlin, nr. 18204619. Cfr. l'aureo RIC I² 121 British Museum, CGR88063. Coniato dalla zecca di Lugdunum. 
nesta gestione della zecca imperiale, cioè simboleggerebbe il valore intrinseco della moneta ${ }^{73}$. È più persuasiva tuttavia l'interpretazione che vede nell'Aequitas la virtù politica della giustizia del principe. In questo senso essa concorre con l'iconografia della Iustitia, della quale si trova una rappresentazione precoce nella monetazione di Tiberio (RIC $\left.\mathrm{I}^{2} 46\right)$, dove ha le sembianze di un busto di donna (Livia?), distinta dalla legenda Iustitia. La Iustitia Augusti compare in una nuova iconografia con Nerva, ad esempio in un denario del 96 (RIC II 6), dove assume le sembianze di una donna a piena figura, seduta, uno scettro nella sinistra e un ramo nella destra ${ }^{74}$ (Fig. 2). Anche questa figurazione - come quella sopra descritta dell'Aequitas - prosegue nella monetazione successiva (in genere al posto del ramo reca una patera sacrificale), pur con minore frequenza rispetto a Aequitas; quasi cessata con Severo Alessandro, la Iustitia si ritrova con Carausio (RIC V 818). Essa rappresenta appunto - sulla scia della valorizzazione augustea della Iustitia - una delle virtù del princeps.

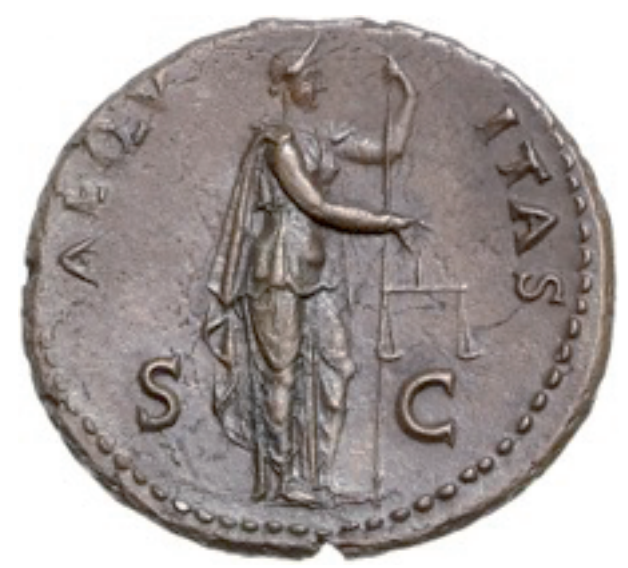

Fig. 1.

73 La posizione di A. Wallace-Hadrill, The emperor and his virtues, in Historia 30 (1981) 298-322, seguita da C.F. Noreña, The communication of the emperor's virtues, in JRS 91 (2001) 146-168, è discussa criticamente da Ostwaldt, Aequitas und Justitia, 27-31; 67-69.

74 Forse con Vespasiano si ha la rappresentazione in piedi, con scettro e coppa: RIC II 11532. 


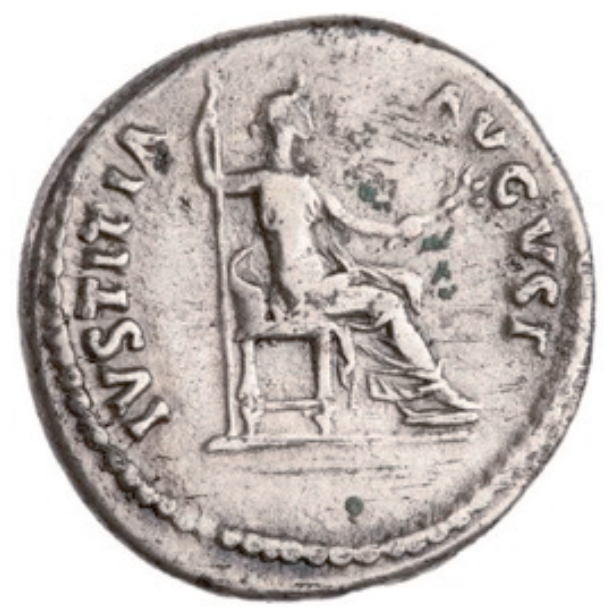

Fig. 2.

La monetazione esibisce dunque una distinzione nitida: Aequitas e Iustitia sono raffigurate in modo sensibilmente diverso, l'una in piedi, l'altra seduta, e solo la prima contraddistinta dalla bilancia.

Questa rappresentazione dell'aequitas non nasce solo con Galba e i Flavi. La donna con la bilancia compare già in una gemma della seconda metà del I secolo a.C.; oltre a reggere la bilancia, porta spighe di grano e un papavero, nell'altra mano una cornucopia ${ }^{75}$; ai suoi piedi, un toro e un ariete. Si tratta di una rappresentazione astrologica della Virgo (Vergine), costellazione la cui stella più luminosa è appunto la Spica (Alfa Virginis); gli animali indicano due costellazioni prossime. La Virgo era protagonista di molti miti. In particolare la si identificava, a partire da Esiodo, con Dike, fuggita dalla terra a causa dell'iniquità degli uomini. Cicerone (in anni non lontani rispetto alla datazione della gemma) tradusse il poema di Arato, che sviluppava con particolare successo il tema, attribuendo alla Virgo il nome di Iustitia (Ovidio le impose infine l'appellativo di Astrea). Tuttavia, il fatto che la Virgo, nella gemma di I secolo a.C., rechi la bilancia (e

75 P. Zazoff, in Antike Gemmen in Deutschen Sammlungen: Berlin, Braunschweig, Göttingen, Hamburg, Hannover, Kassel, München, IV, München, 1968, 79-80, nr. 303; Ostwaldt, Aequitas und Justitia, 31 (riproduzione 257 nr. 11). 
pure la cornucopia) significa appunto che, per chi ha allestito l'immagine, si trattava dell'Aequitas ${ }^{76}$.

Ciò pare confermato da monete coniate in Oriente, sotto Augusto,

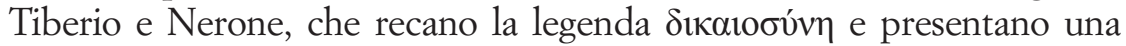
donna con bilancia e cornucopia, appunto i simboli dell'Aequitas: secondo l'interpretazione più attendibile, esse riflettono l'esistenza di un culto romano dell'Aequitas, tradotta con la parola equivalente greca (e non si tratta viceversa di una testimonianza del culto greco della $\delta 1 \kappa \alpha \iota \sigma v ́ v \eta)^{77}$. La cornucopia simboleggia lo stato di benessere, che è il prodotto sociale della aequitas $^{78}$ : vi è insomma la consapevolezza che l'aequitas è una virtù politica, che si accompagna a un assetto dei rapporti interpersonali.

Si potrebbe obiettare alla ricostruzione fin qui seguita di Ostwald (su questo punto costretto dalla scarsità di documenti a ricorrere a ipotesi) che non c'è sicurezza che la gemma tardo-repubblicana (e le stesse monete

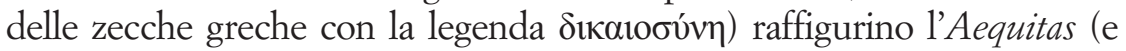
non invece la Iustitia). Tuttavia, l'ipotesi è effettivamente la più probabile: il culto dell'Aequitas è molto risalente (più di quello della Iustitia), come prova la coppa di Vulci dedicata a *Aecetia, databile all'incirca al 280 a.C., intesa nella sua accezione di «corretto comportamento professionale dei negozianti al mercato ${ }^{79}$. Se a ciò si aggiunge che in età flavia la bilancia è tipica dell'Aequitas e la Iustitia ne è priva, le probabilità che la gemma tardo-repubblicana raffiguri la Virgo con le sembianze dell'Aequitas sono in effetti alte (più che supporre una modifica nell'iconografia).

In sintesi: quando appare nelle monete di Galba, l'Aequitas come donna con la bilancia (e cornucopia, o scettro) è probabilmente già una rappresentazione consolidata, attestata almeno dall'ultima età repubblicana.

Quanto alla bilancia, probabilmente fu importata in Grecia come trasformazione del motivo egizio della bilancia che pesa le anime e ne determina il destino ultraterreno (vd. Hom. Il. 22.208-213) ${ }^{80}$. Di qui

76 Ostwaldt, Aequitas und Justitia, 35.

77 Ostwaldt, Aequitas und Justitia, 49-51, dove si respinge l'ipotesi contraria, che

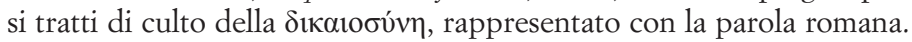

78 Ostwaldt, Aequitas und Justitia, 52.

79 Ostwaldt, Aequitas und Justitia, 65. Vd. supra, p. 39.

80 Cfr. Verg. Aen. 12.725: Iuppiter ipse duas aequato examine lances sustinet et fata inponit diversa duorum («Giove stesso regge i due piatti in equilibrio, e vi pone $\mathrm{i}$ diversi destini dei due»). 
passò a simboleggiare Dike, già in Eschilo (Ag. 249; Choeph. 61). L'adozione a Roma come simbolo dell'aequitas ha tuttavia un presupposto non solo e non tanto nel simbolismo greco, ma anche e soprattutto nella bilancia della mancipatio, atto che comportava (realmente o simbolicamente) la pesatura di metallo come controprestazione ${ }^{81}$. Non è lungo dunque il passo che separa l'A equitas più antica concepita come divinità tutelare del mercato dalla personificazione dell'idea di giustizia basata sull'equilibrio nelle prestazioni (a sua volta prossima all'idea di giustizia commutativa) $)^{82}$.

Anche l'iconografia, insomma, conferma il nocciolo semantico dell'aequum, che consiste nell'equilibrio. Quando Ulpiano esortava il giudice a tenere davanti agli occhi l'aequitas, non faceva che rievocare dunque questo valore, che Modestino, un suo allievo, esprimerà a parole, parlando dell'aequitas che suggerisce una decisione aequa lance ${ }^{83}$.

\section{LA TOPICA DELL'AEQUITAS}

Quando l'aequum romano incontra la sapienza greca ed ellenistica è già una nozione definita e operativa sul piano del diritto, che abbiamo visto confermata anche nell'iconografia. Dall'incontro uscirà però arricchita, di una dimensione molto rilevante.

Nell'ambito della retorica l'aequitas svolge un ruolo argomentati$\mathrm{vo}^{84}$. L'aequum costituisce anzi il punto di riferimento fondamentale della controversia di genere giudiziale, mentre nelle orazioni epidittiche lo è l'onesto e nelle deliberazioni l'utile e - secondo Cicerone - anche l'onesto (Cic. Inv. 2.12): In iudiciis, quid aequum sit, quaeritur; in demonstrationibus, quid honestum; in deliberationibus, ut nos arbitramur, quid bonestum sit et quid utile ${ }^{85}$. La tripartizione ricalca quella che

81 Ostwaldt, Aequitas und Justitia, 61-63.

82 Il cui culto è legato specialmente al mercato: si vedano l'iscrizione EQUI nel tempio di Mercurio di Pompei e il busto proto-bizantino di EQUETAS, discussi da Ostwaldt, Aequitas und Justitia, 63-64.

83 D. 42.1.20: vd. infra, p. 54.

84 Vd. J.-L. Ferrary, Le droit naturel dans les exposés sur les parties du droit des traités de rbétorique, in D. Mantovani - A. Schiavone (a c. di), Testi e problemi del giusnaturalismo romano, Pavia, 2007, 75-94.

85 Cic. Inv. 2.156: Nam placet in iudiciali genere finem esse aequitatem; Part. 98 : 
si legge, ad esempio, in Arist. Rhet. I 3 1358b: aequum corrisponde a

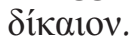

Rispetto al ius, l'aequum (o l'aequum et bonum) svolge un ruolo che è di volta in volta costitutivo (se non v'è una norma applicabile), più spesso interpretativo (quando si tratta di determinare il ius).

Già quando compaiono per la prima volta nei manuali di retorica latina dell'inizio di I secolo a.C., i loci aequitatis - $\mathrm{i}$ luoghi in cui rinvenire gli argomenti per una controversia giudiziale, secondo le modalità dell'inventio - includono però molto più del solo richiamo all'equilibrio. I loci aequitatis si inseriscono in una visione complessiva dell'uomo in società: basti dire che sia nel De inventione sia nei Topica di Cicerone la ricerca dell'aequitas coinvolge la natura e la legislazione positiva, e che per quanto riguarda la natura richiama virtù e valori come religio, pietas, gratia, vindicatio, observantia, veritas. Questo significa che l'aequitas presuppone un'antropologia politica, cioè un'idea dell'uomo nella sua vita in società ${ }^{86}$. Mi pare questo uno snodo centrale del rapporto - complesso e sfuggente, nei punti di contatto e di differenza - fra aequitas e iustitia ${ }^{87}$. Importando la teoria greca della giusti-

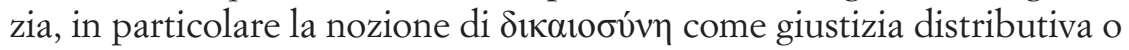
commutativa, i Romani inseriscono l'aequum in uno sfondo più ampio, che ne arricchisce i significati.

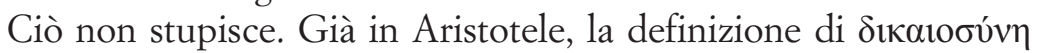
presuppone un'idea ben compiuta dell'uomo e della vita comunitaria, cioè l'innata vocazione dell'uomo a vivere in società. Più in generale, le varie concezioni della giustizia sono «il prodotto di differenti nozioni di

Eius quidem generis finis est aequitas; 100: Nam omnia quae de iure civili aut de aequo et bono disceptantur cadunt in eam formam in qua quale quid sit ambigitur ... quae in aequitate et iure maxime consistit («Infatti tutte le questioni che vengono discusse riguardo al diritto civile e all'equo e buono rientrano nella categoria delle discussioni che vertono sulla qualità, che consiste soprattutto nell'equità e nel diritto»).

86 Questo pensiero, che mi pare suggestivo anche per intendere l'aequitas nel diritto romano e al quale la mia interpretazione deve molto, è espresso a proposito della teoria platonica della giustizia da M. Vegetti, Il problema della giustizia nella Repubblica di Platone, in G.M. Chiodi - R. Gatti (a c. di), La filosofia politica di Platone, Milano, 2008, 27-37.

87 Aequitas come virtù (prossima a iustitia) in Catil. 2.25; cfr. Catil. 4.2; Fin. 2.59; Lael. 82; Off. 1.50; 2.71; De orat. 1.86; Quinct. 10; analisi in Ostwaldt, Aequitas und Justitia, 47. 
società, sullo sfondo di visioni contrastanti riguardo alle necessità naturali e alle opportunità della vita umana. Per comprendere a fondo una concezione della giustizia dobbiamo rendere esplicita l'idea di cooperazione sociale da cui essa deriva» ${ }^{88}$.

Ciò vale appunto per Cicerone, che premette al De inventione un disegno di storia naturale della società, sotto l'angolatura dell'origine e dell'ufficio dell'eloquenza ${ }^{89}$; questa visione sarà poi sviluppata in numerose occasioni, sfruttando in gran parte materiale greco ${ }^{90}$. Non è certo questo il luogo per tracciare un quadro tante volte molto ben studiato. E sufficiente richiamare quel tanto che serve a rendere più esplicita l'interpretazione qui proposta, ossia che l'equità dipende da un'idea di cooperazione sociale, che riesce a dare maggiore precisione al suo significato.

Fra i vari luoghi filosofici, efficace nella sua brevità è Cic. Fin. 5.65: in omni autem bonesto ... nibil est tam illustre nec quod latius pateat quam coniunctio inter homines hominum et quasi quaedam societas et communicatio utilitatum et ipsa caritas generis bumani. Quae nata a primo satu, quod a procreatoribus nati diliguntur et tota domus coniugio et stirpe coniungitur, serpit sensim foras, cognationibus primum, tum affinitatibus, deinde amicitiis, post vicinitatibus, tum civibus et iis, qui publice socii atque amici sunt, deinde totius complexu gentis humanae. Quae animi affectio suum cuique tribuens atque hanc, quam dico, societatem coniunctionis bumanae munifice et aeque tuens iustitia dicitur («Nell'ambito dell'onesto, nulla è tanto illustre e di portata tanto vasta quanto l'unione degli uomini tra loro, una sorta di comproprietà e condivisione delle utilità, e il vincolo d'affetto del genere umano. Esso

88 J. Rawls, Una teoria della giustizia, trad. it., Milano, 1982, 21 ss.

89 Sull'opera, con speciale attenzione proprio alla «sintesi di eloquenza e filosofia abbozzata nel primo proemio» con concetti «tutti praticamente ripresi da elogi greci dell'eloquenza e della cultura», vd. per tutti E. Narducci, Cicerone. La parola e la politica, pref. M. Citroni, Roma-Bari, 2009, 37 ss.

90 Sest. 90-91; De orat. 1.33; Rep. 1.39; Fin. 3.62; Tusc. 1.62; Nat. deor. 2.148; Off. 1.157; 2.73. Vd. per tutti S.E. Smethurst, Cicero and Isocrates, in Trans. Proc. Am. Phil. Assoc. 84 (1953) 262 ss., spec. 275 ss. Per un fine tentativo di legare questi motivi tradizionali alle vicende politiche e biografiche ciceroniane, vd. C. D'Aloja, Legge di natura e lotta politica nell'opera di Cicerone, in D. Mantovani - A. Schiavone (a c. di), Testi e problemi del giusnaturalismo romano, Pavia, 2007, 127 ss., spec. 129 per il De inventione. 
sorge fin dalla nascita, poiché i figli sono amati da chi li ha procreati e l'intera famiglia è unita dal coniugio e dalla discendenza; si diffonde poi a poco a poco al di fuori, dapprima con la parentela, poi con gli affini, poi con le amicizie, poi con il vicinato, poi tramite i cittadini e coloro che sono alleati e amici dello Stato, poi con il genere umano nel suo complesso. L'inclinazione dell'animo di attribuire a ciascuno il suo e che tutela in modo generoso e equo questa consociazione dell'unione umana che sto descrivendo si chiama giustizia»).

La iustitia (intesa esplicitamente alla greca come virtù del suum cuique tribuere) è iscritta in una visione solidaristica della società, che essa contribuisce più di ogni altra virtù a tutelare, ma della quale è anche l'espressione.

Essa si svolge in due principali compiti, non ledere il prossimo e conservare il proprio; a sua volta, si basa sulla fides, cioè sul mantenere la parola data, fondamentale nel rapporto di scambio ${ }^{91}$ (Cic. Off. 1.20): iustitiae primum munus est, ut ne cui quis noceat, nisi lacessitus iniuria, deinde ut communibus pro communibus utatur, privatis ut suis. 21. Sunt autem privata nulla natura, sed aut vetere occupatione, ut qui quondam in vacua venerunt aut victoria, ut qui bello potiti sunt, aut lege, pactione, condicione, sorte (...). Ex quo, quia suum cuiusque fit eorum, quae natura fuerant communia, quod cuique optigit, id quisque teneat; e quo si quis [quaevis] sibi appetet, violabit ius humanae societatis. (...) 23. Fundamentum autem est iustitiae fides, id est dictorum conventorumque constantia et veritas. («il primo compito della giustizia è che uno non nuoccia ad un altro, a meno di essere stato provocato ingiustamente, poi che usi delle cose comuni come comuni, delle cose private come proprie. 21. Non ci sono cose private per natura, ma o per antica occupazione, come i beni di coloro che hanno occupato fondi deserti, o per vittoria, come per i beni conquistati in guerra, o per legge, patto, condizione, sorteggio (...). Ne discende che poiché di quei beni che erano comuni per natura, ciò che tocca a qualcuno diventa suo, costui deve poterlo tenere; e se un altro lo vorrà per sé, violerà il diritto della società

${ }^{91}$ Sul rapporto di scambio come motore per l'emersione del senso di giustizia nelle società primitive, da un punto di vista antropologico, vd. H.Chr. Ehalt, Richtiges Handeln, Gerechtigkeitsvorstellungen und Jurisprudenz - Entwicklungen, Antinomien, Perspektiven, in A. Griesebner - M. Scheutz - H. Weigl (Hrsg.), Justiz und Gerechtigkeit. Historische Beiträge (16.-19. Jahrbundert), Innsbruck, 2002, 19-21. 
umana. Infine, il fondamento della giustizia è la lealtà, ossia la perseveranza e veridicità di ciò che si è detto e convenuto»).

A contatto con la teoria greca della giustizia, l'aequum romano si trova così potenziato: per un verso, esso continua a costituire il criterio fondamentale dell'uguaglianza negli scambi; d'altra parte, si trova inserito in un'antropologia politica: i loci aequitatis si moltiplicano, e li ritroveremo tra poco nei discorsi dei giuristi.

\section{L'AEQUITAS DEI GIURISTI ROMANI}

Per accennare, in modo sintetico, all'aequitas nei giuristi romani, inserendola nella cornice fin qui ricostruita, si deve muovere da un'osservazione lessicale. Nel Digesto la parola iustitia conta 8 occorrenze; aequitas, più di dieci volte tanto ${ }^{22}$. La prima occorrenza di aequitas è in Labeone, ai tempi di Augusto ${ }^{93}$; iustitia compare solo nei giuristi seve-

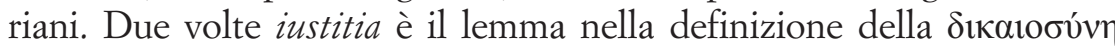
come virtù distributiva, ossia del suum cuique tribuere ${ }^{94}$; mai aequitas ha (almeno esplicitamente) questo ruolo. Accanto ai sostantivi ricorrono gli aggettivi, iustus e aequus (e gli opposti iniustus e iniquus). Iustus ha però spesso un significato che non riguarda la iustitia nel senso di giustizia distributiva o correttiva: innanzitutto viene valorizzato il suo legame con ius, dunque significa legittimo/valido/conforme all'ordinamento (si pensi a iustae nuptiae). In alcune locuzioni (come iustum pretium, che indica il prezzo adeguato) può avvicinarsi a aequus ${ }^{95}$.

Dunque, quando si ha a che fare con la giustizia, la tradizione giuridica preferisce il termine più antico (aequum/aequitas), quello che già era attestato fra III e II secolo nei documenti senatori, nei poeti comici, tragici ed epici e nella prosa catoniana. iustus, 288.

$92 \mathrm{Ne}$ conta 102 occorrenze nelle opere dei giuristi Finkenauer, Iustitia und

93 Citato attendibilmente da Ulp. 38 Ad ed. D. 47.4.1.1; nel I secolo d.C., vd. Sabino e Cassio, in Paul. 6 Ad Plaut. D. 22.1.38.7; Nerat. 3 Membr. D. 6.2.17: Publiciana actio non ideo comparata est, ut res domino auferatur: eiusque rei argumentum est primo aequitas (che mi pare si debba intendere come un'allusione all'aequitas unicuique sum tribuens).

${ }^{94}$ Ulp. 1 Reg. D. 1.1.10 pr.; Tryph. 9 Disp. D. 16.3.31.1.

$95 \mathrm{Su}$ tutti questi significati di iustus, e sulla distinzione rispetto a aequus, vd. 
Questa preferenza lessicale (e la marginalità della iustitia) non implica però l'estraneità rispetto alla teorizzazione della giustizia, quale appare già in atto nei primi trattati di retorica latina a noi pervenuti (inizio I secolo a.C.), di cui abbiamo parlato poco sopra. Anzi, i giuristi sono evidentemente al corrente di questa teorizzazione, che porta a una moltiplicazione (che non significa indistinzione o imprecisione) delle accezioni di equità. Cogliere questa doppia dimensione può aiutare a sbrogliare, almeno in parte, la matassa delle accezioni.

Da una parte, aequitas/aequum continuano a essere impiegati dai giuristi romani nel significato corrispondente al nocciolo semantico, di equaglianza ed equilibrio. Per fare un solo esempio - scelto per la sua plasticità - un giurista di III secolo avanzato, Modestino (2 Diff. D. 42.1.20), ricorre esplicitamente all'immagine dell'aequitas che reca la bilancia per motivare una reciprocità di trattamento: Non tantum dotis nomine maritus in quantum facere possit condemnatur, sed ex aliis quoque contractibus ab uxore iudicio conventus in quantum facere potest, condemnandus est ex divi Pii constitutione. Quod et in persona mulieris aequa lance servari aequitatis suggerit ratio («Non soltanto per la dote il marito viene condannato con il beneficio di competenza, ma anche quando viene convenuto dalla moglie in base ad altri contratti lo si deve condannare nei limiti della sua capacità patrimoniale, secondo una costituzione del divo Antonino Pio. Il che la logica dell'equità suggerisce di applicare con parità di bilancia anche a favore della moglie»).

L'aderenza al nocciolo semantico non impedisce, d'altra parte, ai giuristi romani di ricorrere all'aequitas (e all'aequum) anche in altre accezioni, che dipendono appunto dal suo inserimento in un'idea di società, a sua volta legata a un'idea dell'uomo. I iuris prudentes erano resi familiari a questa elaborazione dalla retorica, che costituiva un elemento di base della loro formazione come di qualsiasi esponente dei ceti dirigenti (anche per chi non avesse poi proseguito gli studi filosofici o si fosse dedicato al diritto).

Finkenauer, Iustitia und iustus, 292-299 (infondato perciò R. Calvo, L'equità nel diritto privato. Individualità, valori e regole nel prisma della contemporaneità, Milano, 2010, 1 nt. 1: «Aequum era dunque un sinonimo di iustum»). La differenza mi pare sancita da un fatto sintattico: iustum è usato, in modo preponderante, in funzione attributiva, cioè come aggettivo che accompagna un sostantivo, in connessioni tendenzialmente fisse (iusta causa, che è la iunctura più frequente; poi iustae nuptiae; iustum matrimonium; iustum pretium; iusta servitus; ecc.). Aequum invece compare in funzione prevalentemente predicativa (aequum est). 
Queste ulteriori sfaccettature assunte dall'aequitas sono però (almeno nella maggior parte) riconducibili a principi e criteri sufficientemente precisi ${ }^{96}$. In altri termini, aequitas/aequum est non è una formula vuota né un veicolo per l'ingresso di valori sociali imprevedibili, o lo è entro limiti che non ne vanificano la capacità di essere percepita come $\operatorname{argomento}{ }^{97}$.

Non è possibile qui tentarne un inventario, di cui vi sono già buoni esempi $^{{ }^{98}}$ (anche se manca ancora una disamina sistematica di tutti i passi, in particolare dei giuristi). Ad esempio, il prolungamento della società oltre al matrimonio e ai figli nella più ampia rete dei consanguinei, che abbiamo letto in Cicerone, è espressa, fra i giuristi, da Gaio, che ne fa derivare la decisione del magistrato giusdicente di estendere la successione a tutti i parenti di sangue, non alla sola linea maschile $(16 \mathrm{Ad}$ ed. prov. D. 38.8.2: hac parte proconsul naturali aequitate motus omnibus cognatis promittit bonorum possessionem, quos sanguinis ratio vocat ad hereditatem, licet iure civili deficiant; «Con questa clausola il proconsole, mosso dall'equità naturale, promette il possesso dei beni ereditari a tutti i parenti, che la logica della consanguineità chiama all'eredità, benché non ne abbiano titolo in base al ius civile») $)^{99}$. È significativo che Gaio parli di

96 Respingendo l'idea di un significato unico, Solidoro Maruotti, Tra morale e diritto, 172-175, ritiene che «aequum e il più tardo vocabolo astratto aequitas assunsero all'incirca otto significati alternativi, di cui sei riconducibili all'età repubblicana e al Principato».

97 In questo senso, è utile, per meglio definire la posizione qui sostenuta, indicare la differenza rispetto a posizioni come quella di Calvo, L'equità nel diritto privato, 2-3: «L'equità compenetra il diritto; il diritto senza equità è come un corpo che non si lascia vibrare dall'anima: il valore sostanziale del diritto è ravvisabile quando si realizzi un ordine sociale giusto. Nel rigore logico imposto dal suddetto finalismo l'antiformalista ravvisa l'autorità sostanziale della legge. Ora la difesa del diritto equo rappresenta un impegno per la libertà assodato che il diritto non è un "ordine cieco", ma è "ordine cosciente", ossia un ordine ancorato ai valori della umanità, tolleranza, coerenza e giustizia». Non so se questa posizione rifletta il valore attuale dell'equità. Per quanto riguarda l'equità romana, essa incorpora a mio avviso un criterio normativo (sufficientemente) preciso, mentre i «valori della umanità, tolleranza, coerenza e giustizia» sono in parte vaghi, in parte estranei all'idea di aequitas (anche se, ovviamente, proprio per la loro vaghezza in parte vi si possono fare rientrare).

98 Mi limito a rinviare a Voci, Ars boni et aequi, 303-306; Stagl, Die Ausgleichung von Vorteil und Nachteil als Inhalt klassischer aequitas, 675-713.

99 Cfr. Ulp. 1 Inst. Coll. 16.9.2; Paul. L. s. de port. D. 48.20 .7 pr. 
naturalis aequitas: fa riferimento a una natura dell'uomo e della società (che è poi quella di Aristotele e degli Stoici), cioè a un'antropologia politica (o se si preferisce a una teoria giusnaturalistica).

Può considerarsi un'estensione di questo modello antropologico l'idea della difesa degli interessi dei minori (attuata dal pretore tramite la restitutio in integrum), che un altro giurista mette sotto lo stesso nome di naturalis aequitas (Ulp. 11 Ad ed. D. 4.4 .1 pr.: hoc edictum praetor naturalem aequitatem secutus proposuit, quo tutelam minorum suscepit; «il pretore ha proposto quest'editto seguendo l'equità naturale, facendosi carico con esso della protezione dei minori»).

S'è visto, sempre in Cicerone, che la giustizia ha due compiti principali (Off. 1.20): sed iustitiae primum munus est, ut ne cui quis noceat, nisi lacessitus iniuria, deinde ut communibus pro communibus utatur, privatis ut suis. Non si tratta, per venire all'equità come criterio di decisione, di pure costruzioni intellettualistiche. Il neminem laedere ha corollari concreti, come il divieto di arricchimento a spese altrui, che a sua volta viene raccolto dai giuristi e posto sotto il segno della natura, ossia appunto di un modello di società legato a una concezione dell'uomo (Pomp. 21 Ad Sab. D. 12.6.14: Nam boc natura aequum est neminem cum alterius detrimento fieri locupletiorem; «Infatti è equo per natura che nessuno si arricchisca a danno di un altro»).

Anche il secondo munus della iustitia, cioè fare avere a ciascuno il suo (che Cicerone specifica precisando che per natura tutto è comune, ma vi è poi l'appropriazione individuale per occupazione di ciò che è deserto, oppure in guerra o per legge o convenzione o assegnazione a sorte $)^{100}$, trova un riflesso nella giurisprudenza. È il pensiero che viene svolto da Nerva, per giustificare la genesi della proprietà dal possesso (Paul. 54 Ad ed. D. 41.2.1.1): dominiumque rerum ex naturali possessione coepisse Nerva filius ait eiusque rei vestigium remanere in his, quae terra mari caeloque capiuntur: nam baec protinus eorum fiunt, qui primi possessionem eorum adprehenderint. Item bello capta et insula in mari enata et gemmae lapilli margaritae in litoribus inventae eius fiunt, qui primus eorum possessionem nanctus est («La proprietà delle cose Nerva figlio sostiene che è sorta dall'impossessamento fisico, e che ne rimane un'impronta negli animali che vengono catturati in terra, mare, cielo:

100 In Cicerone lo stadio prepolitico, rappresentato dall'occupazione, è affiancato a quello politico della guerra e poi della lex e degli scambi. 
infatti divengono immediatamente di coloro che ne hanno per primi preso possesso. Anche le cose catturate in guerra e l'isola nata nel mare e le gemme e i sassi e le perle trovate sui lidi divengono di colui che per primo ne ha ottenuto il possesso»). Dunque il summ cuique tribuere prende qui forma sullo sfondo di una teoria naturale della proprietà.

Ancora: nel modello sociale disegnato da Cicerone (Off. 1.23), il fundamentum iustitiae era individuato nella fides, il rispetto della parola data (che è indispensabile per garantire la compagine sociale attraverso gli scambi). È un principio ripetuto più volte dai giuristi, in particolare da Ulpiano (4 Ad ed. D. 2.14 .1 pr.): buius edicti aequitas naturalis est. Quid enim tam congruum fidei humanae, quam ea quae inter eos placuerunt servare? («l'equità di quest'editto è naturale. Che cosa infatti c'è di più congruo con la lealtà dell'uomo, che mantenere ciò che è stato pattuito fra loro?»); (27 Ad ed. D. 13.5.1): hoc edicto praetor favet naturali aequitati: qui constituta ex consensu facta custodit, quoniam grave est fidem fallere («con quest'editto il pretore favorisce l'equità naturale; egli custodisce gli accordi basati sul consenso, dato che è grave violare la fiducia»).

$\mathrm{Si}$ potrebbe proseguire in questo collegamento fra il disegno "naturalistico" della società e altri valori dell'aequitas che sono stati qui tralasciati. L'esemplificazione è sufficiente per ricomporre un insieme di principi che sono al tempo stesso espressione di tendenze dell'uomo (l'autotutela fisica; l'unione sessuale e la procreazione, da cui discendono come corollari la trasmissione del patrimonio dal padre al figlio e poi ai cognati nonché la difesa dei minori) e regole d'azione indispensabili perché l'uomo possa vivere in società (il divieto di danneggiare l'altro, da cui discende l'equilibrio fra vantaggio e svantaggio; la conservazione di ciò di cui ci si è impossessati; il rispetto della parola data).

$\mathrm{Su}$ questo sfondo, l'aequitas può essere invocata non come uno schema privo di contenuto, e nemmeno da riempire soggettivamente di valori di volta in volta mutevoli ${ }^{101}$, bensì come un criterio normativo, funzionale alla coesione di un concreto modello di società.

D'altra parte (e occorre sottolineare che si tratta di un diverso problema), è difficile dire se il nocciolo semantico di aequum, l'idea di uguaglianza, sia sufficiente a unificare tutte queste accezioni e concre-

101 Benché possa apparire tale la posizione di Talamanca, L'Aequitas, 53-273, l'insigne studioso riconosceva che nell'ambito di un sistema aperto come quello romano (in cui largo spazio è lasciato ai giuristi nella determinazione del ius) l'aequitas «non 
tizzazioni dell'idea di aequum o se invece l'aequitas sia diventata il simbolo unificante di una serie di principi almeno in parte slegati dal suo nucleo semantico iniziale e discendenti invece dal più volte ricordato modello sociale elaborato nella filosofia greca e importato - specialmente tramite la retorica - a Roma. Insomma, anche per Roma può valere chiedersi «quante equità» vi fossero.

Lasciando aperto il problema, possiamo concludere il percorso con la lettura di un brano in cui il nocciolo dell'uguaglianza e il modello sociale sembrano incontrarsi. Il brano è tratto dal commento Ad edictum di Giulio Paolo, prolifico giurista e uomo politico, che raggiunse probabilmente la carica di praefectus praetorio sotto Elagabalo o Severo Alessandro, nella prima metà del III secolo d.C.; il passo, nell'opera di Paolo, si inseriva nel contesto dell'actio locati conducti, sotto cui ricadevano anche i contratti che oggi qualificheremmo di trasporto (Paul. 34 Ad ed. D. 14.2.2 pr.): Si laborante nave iactus factus est, amissarum mercium domini, si merces vehendas locaverant, ex locato cum magistro navis agere debent: is deinde cum reliquis, quorum merces salvae sunt, ex conducto, ut detrimentum pro portione communicetur, agere potest (...): aequissimum enim est commune detrimentum fieri eorum, qui propter amissas res aliorum consecuti sunt, ut merces suas salvas baberent ${ }^{102}$ («Siccome la nave era in difficoltà, parte del carico fu gettata in mare: $\mathrm{i}$ proprietari delle merci andate perdute, se avevano locato le merci perché fossero trasportate, devono agire in base al contratto di locazione

può, come nei sistemi chiusi, avere la funzione formale di strumento che veicola nell'ambito dell'ordinamento giudizi di valore in quello non sanciti» (p. 59). Vi erano «limiti intrinseci all'operare dei giureconsulti, che derivavano dalla consapevolezza ... di non essere dei legislatori, ma soltanto dei mediatori dell'ordine insito nella natura stessa dei rapporti ... Con ciò essi subivano un condizionamento di tipo giusnaturalistico». Anche sotto un altro profilo l'indagine dell'A. mi pare offra un quadro del tutto condivisibile. Egli constata che nelle costituzioni degli imperatori fra la morte di Alessandro Severo e Diocleziano il richiamo all'aequitas/aequum non serve, nella maggior parte dei casi, a giustificare soluzioni innovative, bensì a confermare soluzioni già accettate. Questo risultato sottolinea che - a differenza di quanto avviene nei sistemi di diritto codificato - un sistema come quello romanistico tendeva a vedere l'aequum non come un contrappunto al ius, bensì come un suo valore costitutivo.

102 Nella parte omessa del testo, si legge un responso del giurista repubblicano Servio Sulpicio Rufo che prefigura la soluzione proposta da Paolo; inoltre si esamina il caso di una conduzione parziale della nave da trasporto, diverso da quello di locatio 
contro il comandante della nave: costui può poi agire contro gli altri, le cui merci sono salve, in base all'azione di conduzione, in modo che il danno sia condiviso proporzionalmente (...): è infatti equissimo che il danno diventi comune di quelli che grazie alla perdita delle cose altrui sono riusciti ad avere salve le loro merci» $)^{103}$.

Per uscire dalla tempesta, una nave è stata alleggerita di parte del carico; giunta all'approdo, i proprietari delle merci salve devono contribuire proporzionalmente a indennizzare i proprietari delle merci gettate fuori bordo. Fra la perdita degli uni e il vantaggio degli altri c'è infatti un nesso di causalità (propter amissas res aliorum consecuti sunt, ut merces suas salvas haberent); esso impone di considerare la situazione degli uni in rapporto a quella degli altri; lo squilibrio è evidente. Per evitarlo, aequissimum (...) est commune detrimentum fieri. L'equità è qui criterio di decisione, che persegue l'equilibrio fra le parti di un rapporto, evitando l'arricchimento dell'una a detrimento dell'altra. Si tratta del criterio della giustizia correttiva (aritmetica). Il criterio equitativo è a tal punto operativo (non dunque un'idea vaga) che serve anche come criterio di calcolo. Per stabilire il contributo, si procedeva alla somma

mercium vehendarum oggetto della prima parte del testo (sui diversi assetti di interesse della locazione marittima, vd. R. Fiori, La definizione della Locatio conductio: giurisprudenza romana e tradizione romanistica, Napoli, 1999, 128-155).

103 Su questo testo vd. l'eccellente esegesi di D. Schanbacher, Zur Rezeption und Entwicklung des rhodischen Seewurfrechts in Rom, in Humaniora: Medizin - Recht Geschichte. Festschrift A. Laufs, Heidelberg, 2006, 257-273. Nell'interpretare il testo, e ricondurlo all'aequitas, sono opportune alcune precisazioni. La decisione sfrutta l'inquadramento del trasporto nel contratto di locatio et conductio e utilizza l'esistenza del rapporto fra ciascuno dei proprietari delle merci con il comandante, per ottenere una sorta di società fra tutti. L'actio locati et conducti era un iudicium bonae fidei, che attribuiva al giudice di stabilire le obbligazioni reciproche, quidquid alterum alteri dare facere praestare oportet ex fide bona. Il riferimento alla fides bona permette al giudice (e al giurista Paolo che ne prefigura la decisione) una libertà di apprezzamento che in altri rapporti non avrebbe avuto. Ancora: nel caso specifico, la fides bona viene concretizzata dall'aequitas, intesa come equilibrio patrimoniale, che a sua volta coincide con la giustizia in senso correttivo (commutativo). Infine, mettere in comune l'avaria era un principio già accolto nel commercio marittimo e ascritto a una lex Rhodia de iactu (variamente identificata dagli studiosi con un'usanza commerciale o una vera e propria legge di Rodi). In sintesi: il principio della cd. lex Rhodia, applicato nel commercio marittimo, che è un'applicazione della giustizia correttiva, cioè dell'aequitas, trova la sua realizzazione sul piano dell'ordinamento romano grazie al iudicium bonae fidei. 
ideale del valore di tutti i beni, salvati e perduti, e si procedeva alla ripartizione in modo che ciascuno partecipasse nella medesima percentuale al danno (es. se un proprietario aveva avuto salvo 100 su 100 e l'altro, su 100, aveva perduto 60, il proprietario delle merci salve doveva versare 30 , in modo che ciascuno dei due risultasse avere salvato $70 \mathrm{e}$ perduto 30). È un criterio che si applica anche al proprietario della nave, se ha dovuto sacrificarne l'albero per proseguire la navigazione: anche in questo caso avrà luogo l'aequitas contributionis, cioè «la parità nella ripartizione del danno», come spiega Ermogeniano, uno degli ultimi giuristi di cui sia rimasta la testimonianza letteraria, all'epoca di Diocleziano (2 Iuris ep. D. 14.2.5.1: Arbore caesa, ut navis cum mercibus liberari possit, aequitas contributionis babebit locum $)^{104}$.

Di fronte a un problema concreto, i giuristi romani trovano nell'aequum un criterio sufficientemente preciso di risoluzione del conflitto di interessi. È una logica che pare molto risalente nella mentalità romana $\mathrm{e}$ che trova nell'attributo iconografico della bilancia la sua figurazione. $\mathrm{Ma}$ il passo di Paolo lascia intravedere un contorno più ampio. La nave è facile metafora di un gruppo sociale, nel quale la situazione dell'uno è legata a quella dell'altro ${ }^{105}$. Anche senza passare per una teorizzazione più sofisticata, il caso dell'avaria comune permette di vedere che anche nel suo nucleo fondamentale l'equità come equilibrio è legata a un modello di società, a un'idea di coordinazione nei rapporti umani. La vaghezza che le si rimprovera dipende forse dal considerarla isolata dal modello in cui si inseriva, e da cui dipende la sua giuridicità. Per capire che cosa sia aequum, occorre comprendere in che modo l'interprete considera l'uomo nel rapporto con gli altri in società. Lo stesso vale nel diritto romano: l'aequitas presuppone un'antropologia politica, cioè un'idea di che cosa sia l'uomo considerato individualmente e nelle sue relazioni sociali.

104 Sul brano Talamanca, L'Aequitas, 256-262, dove anche la proposta di traduzione.

105 Sull'attività di governo come guida della nave vd. Cambiano, Come nave in tempesta, spec. 108-132. 